BULLETIN (New Series) OF THE

AMERICAN MATHEMATICAL SOCIETY

Volume 49, Number 4, October 2012, Pages 475-506

S 0273-0979(2012)01383-6

Article electronically published on July 3, 2012

\title{
NOTES ON TOPOLOGICAL STABILITY
}

\author{
JOHN MATHER
}

\section{ForewORD TO THE 1970 BOOKLET}

These notes are part of the first chapter of a series of lectures given by the author in the spring of 1970. The ultimate aim of these notes will be to prove the theorem that the set of topologically stable mappings form a dense subset of $C^{\infty}(N, P)$ for any finite dimensional manifolds $N$ and $P$ where $N$ is compact. The first chapter is a study of Thom-Whitney theory of stratified sets and stratified mappings. The connection of the material in these notes with the theorem on the density of topologically stable mappings appears in Section [11, where we give Thom's second isotopy lemma. This result gives sufficient conditions for two mappings to be topologically equivalent.

\section{LIST OF REVISIONS}

A List of Revisions to the 1970 booklet is appended at the end of this article.

\section{ACKNOWLEDGMENTS}

The ideas explained in these Notes are due to René Thom. The results are either variants on results of Thom or known results, which the reader needs to know to understand Thom's results, but which Thom did not explain in his articles on this subject.

The original version of these notes was typed by Mary McQuillen and made into a booklet by the Harvard printing office in 1970. This revised version of the notes was typeset in $\mathcal{A} \mathcal{M} \mathcal{S}$-LATEX by Shu Otsuka in December 2011. I wish to thank Susan Friedlander and Mark Goresky for inviting me to publish these notes in the Bulletin of the American Mathematical Society. My work on the 1970 booklet was supported by an NSF grant. My work on this revision was supported by The Ambrose Monell Foundation.

\section{Contents}

Foreword to the 1970 Booklet 475

List of Revisions

Acknowledgments

1. Condition $a \quad 476$

2. Condition $b \quad 476$

3. Blowing up 478

4. An intrinsic formulation of condition $b \quad 480$

5. Whitney stratifications

6. Tubular neighborhoods 481 
7. Control data

8. Abstract stratified sets

9. Controlled vector fields 493

10. One parameter groups 495

11. The isotopy lemmas of Thom 499

References

List of Revisions

\section{Condition $a$}

We begin by introducing some notions that are due to Whitney ([5] and [6]).

Let $\mu$ be a positive integer or $\infty$, which will be fixed throughout this paper. By "smooth" we will mean differentiable of class $C^{\mu}$.

Let $M$ be a smooth (i.e., $\left.C^{\mu}\right) n$-manifold without boundary. By a smooth (i.e., $C^{\mu}$ ) submanifold of $M$, we will mean a subset $X$ of $M$ such that for every $x \in X$ there exists a coordinate chart $(\varphi, U)$ of class $C^{\mu}$ such that $x \in U$ and $\varphi(X \cap U)=\mathbb{R}^{k} \cap \varphi(U)$, for a suitable coordinate plane $\mathbb{R}^{k}$ in $\mathbb{R}^{n}$. In the definition of submanifold, we do not assume that $X$ is closed. However, it follows from the definition of submanifold that $X$ is locally closed, i.e., each point in $X$ has a neighborhood $U$ in $M$ such that $X \cap U$ is closed in $U$.

If $X$ is an $r$-dimensional submanifold of $M$ and $x \in X$, then the tangent space $T X_{x}$ of $X$ at $x$ is a point in the Grassmannian bundle of $r$-planes in $T M_{x}$. In what follows convergence means convergence in the standard topology of this bundle.

Let $X$ and $Y$ be smooth submanifolds of $M$ and let $y \in Y$. Set $r=\operatorname{dim} X$.

Definition 1.1. We say the pair $(X, Y)$ satisfies condition $a$ at $y$ if the following holds. Given any sequence $\left\{x_{i}\right\}$ of points in $X$ such that $x_{i} \rightarrow y$ and $T X_{x_{i}}$ converges to some $r$-plane $\tau \subseteq T M_{y}$, we have $T Y_{y} \subseteq \tau$.

Example 1.2 (Whitney [6]). Let $x, y, z$ denote coordinates for $\mathbb{C}^{3}$. Let $Y$ be the $z$-axis and let $X$ be the set $\left\{z x^{2}-y^{2}=0\right\}$ with the $z$-axis deleted. Then $X$ and $Y$ are complex analytic submanifolds of $\mathbb{C}^{3}$. It is easily seen that $(X, Y)$ satisfies condition $a$ at all points of $Y$ except the origin, and that it does not satisfy condition $a$ there.

We will say that the pair $(X, Y)$ satisfies condition $a$ if it satisfies condition $a$ at every point of $Y$.

In Example 1.2, the pair $(X, Y)$ does not satisfy condition $a$. If we set $Z=\{0\}$ and $Y^{\prime}=Y-Z$, then the pairs $\left(X, Y^{\prime}\right),(X, Z)$, and $\left(Y^{\prime}, Z\right)$ do satisfy condition $a$.

\section{Condition $b$}

We will begin by defining Whitney's condition $b$ for submanifolds of $\mathbb{R}^{n}$. Then we extend this definition to submanifolds of an arbitrary manifold, using the definition in $\mathbb{R}^{n}$. We will also show that condition $b$ implies condition $a$.

If $x, y \in \mathbb{R}^{n}$ and $x \neq y$, then the secant $\widehat{x y}$ will denote the line in $\mathbb{R}^{n}$ which is parallel to the line joining $x$ and $y$ and passes through the origin. For any $x \in \mathbb{R}^{n}$ we identify $T_{x} \mathbb{R}^{n}$ with $\mathbb{R}^{n}$ in the standard way.

Let $X, Y$ be (smooth) submanifolds of $\mathbb{R}^{n}$. Let $y \in Y$. Let $r=\operatorname{dim} X$. 
Definition 2.1. We say that the pair $(X, Y)$ satisfies condition $b$ at $y$ if the following holds. Let $\left\{x_{i}\right\}$ be a sequence of points in $X$, converging to $y$ and $\left\{y_{i}\right\}$ a sequence of points in $Y$, also converging to $y$. Suppose $T X_{x_{i}}$ converges to some $r$ plane $\tau \subseteq \mathbb{R}^{n}$ and that $x_{i} \neq y_{i}$ for all $i$ and the secants $\widehat{x}_{i} y_{i}$ converge (in projective space $\mathbb{P}^{n-1}$ ) to some line $\ell \subseteq \mathbb{R}^{n}$. Then $\ell \subseteq \tau$.

Let $\left(X^{\prime}, Y^{\prime}\right)$ be a second pair of submanifolds of $\mathbb{R}^{n}$, and let $y^{\prime} \in Y^{\prime}$.

Lemma 2.2. Suppose there exist open neighborhoods $U$ and $U^{\prime}$ of $y$ and $y^{\prime}$ in $\mathbb{R}^{n}$ and a (smooth) diffeomorphism $\varphi: U \rightarrow U^{\prime}$ such that $\varphi(U \cap X)=U^{\prime} \cap X^{\prime}$, $\varphi(U \cap Y)=U^{\prime} \cap Y^{\prime}$ and $\varphi(y)=y^{\prime}$. Then $(X, Y)$ satisfies condition $b$ at $y$ if and only if $\left(X^{\prime}, Y^{\prime}\right)$ satisfies condition $b$ at $y^{\prime}$.

Proof. Obvious.

Definition 2.3. Let $M$ be a manifold and $X, Y$ submanifolds. Let $y \in Y$. We say that $(X, Y)$ satisfies condition $b$ at $y$ if for some coordinate chart $(\varphi, U)$ about $y$, we have that the pair $(\varphi(U \cap X), \varphi(U \cap Y))$ satisfies condition $b$ at $\varphi(y)$.

In view of Lemma 2.2 if $(X, Y)$ satisfies condition $b$ at $y$, then for every coordinate chart $(\varphi, U)$ about $y$, we have that $(\varphi(U \cap X), \varphi(U \cap Y))$ satisfies condition $b$ at $y$.

For the rest of this section, let $M$ be a manifold and $X$ and $Y$ submanifolds and let $y \in Y$.

Proposition 2.4. If $(X, Y)$ satisfies condition $b$ at $y$, then it satisfies condition a at $y$.

Proof. Since both conditions $a$ and $b$ are purely local, we may suppose that $X$ and $Y$ are submanifolds of $\mathbb{R}^{n}$. Let $\left\{x_{i}\right\}$ be a sequence of points in $X$ such that $x_{i} \rightarrow y$ and $T X_{x_{i}} \rightarrow \tau$, for some $\tau \subseteq T \mathbb{R}_{y}^{n}=\mathbb{R}^{n}$. We must show that $T Y_{y} \subseteq \tau$. Suppose otherwise. Then there exists a line $\ell \subseteq \mathbb{R}^{n}$, passing through the origin, such that $\ell \subseteq T Y_{y}$ but $\ell \nsubseteq \tau$. Since $\ell \subseteq T Y_{y}$, we can choose a sequence of points $\left\{y_{i}\right\}$ in $Y$ such that $y_{i} \neq x_{i}, y_{i} \rightarrow y$ and $\widehat{x}_{i} y_{i} \rightarrow \ell$. But since $\ell \nsubseteq \tau$, this contradicts condition $b$.

We say $(X, Y)$ satisfies condition $b$ if it satisfies condition $b$ at every point $y \in Y$.

Example 2.5. Let $X$ be the spiral in $\mathbb{R}^{2}$ defined by the condition that the tangent of $X$ makes a constant angle with the radial vector, and let $Y$ be the origin. In polar coordinates, this spiral is given by $r-\beta \theta=$ constant. Then the pair $(X, Y)$ does not satisfy condition $b$. For, by definition, the angle $\alpha$ between the line $T X_{x}$ and the secant $\widetilde{0 x}$ is independent of $x$. If $\left\{x_{i}\right\} \subset X$ is a sequence converging to 0 and the tangents $\left\{T X_{x_{i}}\right\}$ converge to a line $\tau \subseteq \mathbb{R}^{2}$, then $\widehat{0 x_{i}}$ converges to a line $\ell$, which makes an angle $\alpha$ with $\tau$.

Example 2.6 (Whitney [6]). Let $x, y, z$ be coordinates for $\mathbb{C}^{3}$. Let $Y$ be the $z$-axis. Let $X$ be the set $\left\{y^{2}+x^{3}-z^{2} x^{2}=0\right\}$ with the $z$-axis deleted. It is easily seen that the pair $(X, Y)$ satisfies condition $a$, and the pair $(X, Y)$ satisfies condition $b$ at all points of $Y$ except the origin and that it does not satisfy condition $b$ there.

Proposition 2.7. Suppose $y \in \overline{X-Y}$ and $(X, Y)$ satisfies condition $b$ at $y$. Then $\operatorname{dim} Y<\operatorname{dim} X$. 
Proof. It is enough to consider the case when $M=\mathbb{R}^{m}$. Since $y \in \overline{X-Y}$, there exists a sequence $\left\{x_{i}\right\}$ in $X-Y$ which converges to $y$. By the compactness of the Grassmannian, we may suppose, by passing to a subsequence if necessary, that $\left\{T X_{x_{i}}\right\}$ converges to an $r$ plane $\tau \subseteq \mathbb{R}^{m}$ (where $r=\operatorname{dim} X$ ). Since condition $b$ implies condition $a$ (Proposition 2.4), $T Y_{y} \subseteq \tau$. For $i$ sufficiently large, there is a point $y_{i}$ on $Y$ which minimizes the distance to $x_{i}$. By passing to a subsequence if necessary, we may suppose the secants $x_{i} y_{i}$ converge to a line $\ell \subseteq \mathbb{R}^{n}$. Since $y_{i}$ minimizes the distance to $x_{i}$, the secant $\widehat{x_{i} y_{i}}$ is orthogonal to $T Y_{y_{i}}$; hence $\ell$ is orthogonal to $T Y_{y}$. Since $(X, Y)$ satisfies condition $b$ at $y$, we have $\ell \subseteq \tau$. We have shown $T Y_{y}+\ell \subseteq \tau$ and $\ell$ is orthogonal to $T Y_{y}$; hence $\operatorname{dim} X=\operatorname{dim} \tau>\operatorname{dim} T Y_{y}=$ $\operatorname{dim} Y$.

\section{BLOWING UP}

In the next section, we will give an intrinsic formulation of condition $b$ which will be useful later on. This formulation depends on the notion of blowing up a manifold along a submanifold, which we define in this section.

Let $N$ be a manifold and $U$ a closed submanifold. By the manifold $B_{U} N$ obtained by blowing up $N$ along $U$, we will mean the manifold defined in the following way. As a set $B_{U} N$ is the disjoint union $(N-U) \cup P \eta_{U}$, where $P \eta_{U}$ denotes the projective normal bundle of $U$ in $N$.

By the natural projection $\pi: B_{U} N \rightarrow N$, we mean the mapping defined by letting $\pi \mid P \eta_{U}$ be the projection of $P \eta_{U}$ on $U$ and letting $\pi \mid N-U$ be the inclusion of $N-U$ into $N$.

To define the differentiable structure on $B_{U} N$, we first consider the case when $N$ is open in $\mathbb{R}^{n}$ and $U=\mathbb{R}^{r} \cap N$, where $\mathbb{R}^{r}$ is the coordinate plane defined by the vanishing of the last $n-r$ coordinates. Then we have a mapping $\alpha: B_{U} N \rightarrow$ $\mathbb{R}^{n} \times \mathbb{R} P^{n-r-1}$ defined as follows. First, $\alpha \mid P \eta_{U}$ is the standard identification of $P \eta_{U}$ with $U \times \mathbb{R} P^{n-r-1} \subseteq \mathbb{R}^{n} \times \mathbb{R} P^{n-r-1}$. Secondly, if $x=\left(x_{1}, \cdots, x_{n}\right) \in \mathbb{R}^{n}-\mathbb{R}^{r}$, then $\alpha(x)=(x, \beta(x))$, where $\beta(x)$ is the point in $\mathbb{R} P^{n-r-1}$ with homogeneous coordinates $\left(x_{r+1}, \cdots, x_{n}\right)$.

It is easily verified that $\alpha\left[B_{U} N\right]$ is a $C^{\infty}$ submanifold of $\mathbb{R}^{n} \times \mathbb{R} P^{n-r-1}$, as follows. Let $\left(x_{1}, \cdots, x_{n}\right)$ denote the coordinates of $\mathbb{R}^{n}$. Let $X_{r+1}, \cdots, X_{n}$ denote the homogeneous coordinates for $\mathbb{R} P^{n-r-1}$. For $r+1 \leq i \leq n$, let $Z_{i}$ denote the subset of $\mathbb{R} P^{n-r-1}$ defined by $X_{i} \neq 0$, and let $X_{j i}$ be the real valued function $X_{j i}=X_{j} / X_{i}$ on $Z_{i}$. Then the intersection of $\alpha\left[B_{U} N\right]$ with $N \times Z_{i}$ is the set defined by

$$
x_{j}=X_{j i} x_{i} \quad r+1 \leq j \leq n, \quad j \neq i .
$$

Therefore $\alpha\left[B_{U} N\right]$ is a submanifold of $\mathbb{R}^{n} \times \mathbb{R} P^{n-r-1}$.

Since the mapping $\alpha$ is injective, we may define a manifold structure on $B_{U} N$ by pulling back the manifold structure on $\alpha\left[B_{U} N\right]$.

Now, let $N^{\prime}$ be a second open subset of $\mathbb{R}^{n}$, let $U^{\prime}=\mathbb{R}^{r} \cap N^{\prime}$, and let $\varphi:(N, U) \rightarrow$ $\left(N^{\prime}, U^{\prime}\right)$ be a $C^{\mu}$ diffeomorphism. Let $\varphi_{*}: B_{U} N \rightarrow B_{U^{\prime}} N^{\prime}$ be the induced mapping, defined by letting $\varphi_{*} \mid P \eta_{U}: P \eta_{U} \rightarrow P \eta_{U^{\prime}}$ be the mapping induced by the differential, and letting $\varphi_{*} \mid(N-U): N-U \rightarrow N^{\prime}-U^{\prime}$ be the restriction of $\varphi$. Then $\varphi_{*}$ is a diffeomorphism of class $C^{\mu-1}$.

To show this, we first observe that $\varphi_{*}$ is a bijection and $\left(\varphi_{*}\right)^{-1}=\left(\varphi^{-1}\right)_{*}$. Therefore, it suffices to show that $\varphi_{*}$ is of class $C^{\mu-1}$. To show this, it is enough 
to show that $x_{i} \circ \varphi_{*}$ is of class $C^{\mu-1}, 1 \leq i \leq n$, that $\varphi_{*}^{-1} Z_{i}$ is open, $r+1 \leq i \leq n$, and that $X_{j i} \varphi_{*}$ is of class $C^{\mu-1}$ for $r+1 \leq j \leq n$ and $j \neq i$. Since

$$
x_{i} \circ \varphi_{*}=x_{i} \circ \varphi \circ \pi,
$$

where $\pi: B_{U} N \rightarrow N$ is the natural projection, the first statement is obvious.

To prove the remaining two statements, we set $\varphi_{i}=x_{i} \circ \varphi$ and observe that there exist functions $\psi_{i \alpha}$ of class $C^{\mu-1}$, for $r+1 \leq i, \alpha \leq n$, such that

$$
\varphi_{i}=\sum_{\alpha=r+1}^{n} x_{\alpha} \psi_{i \alpha} .
$$

This is proved as follows. Since for $r+1 \leq i \leq n$, we have that $\varphi_{i}$ vanishes on $U=N \cap \mathbb{R}^{r}$, we get that

$$
\begin{aligned}
\varphi_{i}\left(x_{1}, \cdots, x_{n}\right) & =\int_{0}^{1} \frac{d}{d t} \varphi_{i}\left(x_{1}, \cdots, x_{r}, t x_{r+1}, \cdots, t x_{n}\right) d t \\
& =\sum_{\alpha=r+1}^{n} x_{\alpha} \int_{0}^{1} \frac{\partial \varphi_{i}}{\partial x_{\alpha}}\left(x_{1}, \cdots, x_{r}, t x_{r+1}, \cdots, t x_{n}\right) d t
\end{aligned}
$$

so that (3.1) holds, where

$$
\psi_{i \alpha}=\int_{0}^{1} \frac{\partial \varphi_{i}}{\partial x_{\alpha}}\left(x_{1}, \cdots, x_{r}, t x_{r+1}, \cdots, t x_{n}\right) d t .
$$

In view of (3.1), $\varphi_{*}^{-1}\left(Z_{i}\right) \cap Z_{k}$ is the subset of $Z_{k}$ defined by

$$
\sum_{\alpha=r+1}^{n} X_{\alpha k} \psi_{i \alpha} \neq 0,
$$

and hence is open. It follows that $\varphi_{*}^{-1} Z_{i}$ is open. It also follows from (3.1) that

$$
X_{j i} \circ \varphi_{*}=\frac{\sum_{\alpha=r+1}^{n} X_{\alpha k} \psi_{j \alpha}}{\sum_{\alpha=r+1}^{n} X_{\alpha k} \psi_{i \alpha}}
$$

on $\varphi_{*}^{-1}\left(Z_{i}\right) \cap Z_{k}$, and hence is of class $C^{\mu-1}$ there.

This completes the proof that $\varphi_{*}$ is a diffeomorphism of class $C^{\mu-1}$.

Now we return to the general situation where $N$ is a manifold, and $U$ is a closed submanifold, both of class $C^{\mu}$. In view of what we have just done, we can construct a differentiable structure on the part of $B_{U} N$ which lies above any coordinate patch, and the differentiable structures above different coordinate patches are $C^{\mu-1}$ compatible. Thus, we obtain the structure of a manifold of class $C^{\mu-1}$ on $B_{U} N$.

Note that the natural projection $\pi: B_{U} N \rightarrow N$ is differentiable of class $C^{\mu-1}$.

Since we have defined a structure of a manifold of class $C^{\mu-1}$ on $B_{U} N$, we have also defined a topology on $B_{U} N$. In the local case, when $N=\mathbb{R}^{n}$ and $U=\mathbb{R}^{r}$, this topology may be described more directly. Let $\left\{x_{i}\right\}$ be a sequence of points in $\mathbb{R}^{n}-\mathbb{R}^{r}$, and suppose $x_{i} \rightarrow x \in \mathbb{R}^{r}$. Let $\ell \in \mathbb{R} P^{n-r-1}$, so that $(x, \ell)$ is a member of $B_{U} N$, if we identify $B_{U} N$ with the subset $\alpha\left(B_{U} N\right)$ of $\mathbb{R}^{n} \times \mathbb{R} P^{n-r}$, as above. Then it is easily seen that $\left\{x_{i}\right\}$ converges (in $B_{U} N$ ) to $(x, \ell)$ if and only if the secants $\widehat{x_{i} x_{i}^{\prime}}$ converge to $\ell$, where $x_{i}^{\prime}$ denotes the projection of $x_{i}$ on $\mathbb{R}^{r}$.

This suggests that it should be possible to reformulate condition $b$ in terms of "blowing up." We do this in the next section. 


\section{An INTRINSiC FORMULATION OF CONDITION $b$}

Let $N$ be a smooth manifold. Let $\Delta_{N}$ denote the diagonal in $N^{2}$. By the fat square of $N$, we mean the manifold $F(N)$ obtained by blowing up $N^{2}$ along $\Delta_{N}$.

The normal bundle $\eta$ of $\Delta_{N}$ in $N^{2}$ can be identified with the tangent bundle $T N$ in a canonical way, as follows. If $x \in \Delta_{N}$, then by definition

$$
\eta_{x}=\left(T N_{x} \oplus T N_{x}\right) / \text { diagonal. }
$$

The mapping of $T N_{x} \oplus T N_{x}$ into $T N_{x}$ which sends $v \oplus w$ to $v-w$ induces an isomorphism of $\eta_{x}$ with $T N_{x}$. We use this isomorphism to identify $\eta_{x}$ with $T N_{x}$.

From this identification and the definition of the process of blowing up a manifold along a submanifold, it follows that

$$
F(N)=P T(N) \cup\left(N^{2}-\Delta_{N}\right) \quad(\text { disjoint union) }
$$

where $P T(N)$ denotes the projective tangent bundle of $N$. Thus, points of $F(N)$ are of two kinds: pairs $(x, y)$ with $x, y \in N$ and $x \neq y$ and tangent directions on $N$.

It follows from the previous section that $F(N)$ is a manifold of class $C^{\mu-1}$.

Roughly speaking, a sequence $\left\{\left(x_{i}, y_{i}\right)\right\}$ of points in $N^{2}-\Delta_{N}$ converges to a tangent direction $\ell$ on $N$ if the sequences $\left\{x_{i}\right\}$ and $\left\{y_{i}\right\}$ converge to the same point $x$ in $N$ and the direction from $x_{i}$ to $y_{i}$ converge to $\ell$. In the case $N=\mathbb{R}^{n}$, this can be made precise: $\left\{\left(x_{i}, y_{i}\right)\right\}$ converges to $(x, \ell) \in \mathbb{R}^{n} \times \mathbb{R} P^{n-1}$ if both $\left\{x_{i}\right\}$ and $\left\{y_{i}\right\}$ converge to $x$, and the secants $\widehat{x_{i} y_{i}}$ converge to $\ell$.

Now let $X$ and $Y$ be smooth submanifolds of $N$ and let $y \in Y$. Suppose $\mathrm{Y}$ is closed. In view of the previous paragraph, we obtain the following result.

Proposition 4.1. The pair $(X, Y)$ satisfies condition $b$ at $y$ if and only if the following condition holds. Let $\left\{x_{i}\right\}$ be any sequence of points in $X$ and $\left\{y_{i}\right\}$ any sequence of points in $Y$ such that $x_{i} \neq y_{i}$. Suppose $\left\{x_{i}\right\} \rightarrow y,\left\{y_{i}\right\} \rightarrow y,\left\{\left(x_{i}, y_{i}\right)\right\}$ converges to a line $\ell \subseteq P T N_{y}$, and $\left\{T X_{x_{i}}\right\}$ converges (in the Grassmannian of $r$-planes in $T N$, where $r=\operatorname{dim} X)$ to an $r$-plane $\tau \subseteq T N_{y}$. Then $\ell \subseteq \tau$.

\section{WhitNEY STRATIFICATIONS}

Let $M$ be a smooth (i.e., $C^{\mu}$ ) manifold without boundary. Let $S$ be a subset of $M$. By a stratification $\mathcal{S}$ of $S$, we will mean a cover of $S$ by pairwise disjoint smooth submanifolds of $M$, which lie in $S$. We will say that $\mathcal{S}$ is locally finite if each point of $M$ has a neighborhood which meets at most finitely many strata. We say $\mathcal{S}$ satisfies the condition of the frontier if for each stratum $X$ of $\mathcal{S}$ its frontier $(\bar{X}-X) \cap S$ is a union of strata.

We will say $\mathcal{S}$ is a Whitney stratification if it is locally finite, satisfies the condition of the frontier, and $(X, Y)$ satisfies condition $b$ for any pair $(X, Y)$ of strata of $\mathcal{S}$.

Let $\mathcal{S}$ be a Whitney stratification of a subset $S$ of a manifold $M$. Suppose $X$ and $Y$ are strata. We write $Y<X$ if $Y$ is in the frontier of $X$. In view of Proposition 2.7. if $Y<X$ then $\operatorname{dim} Y<\operatorname{dim} X$. It follows easily that the relation " $<$ " defines a partial order on $\mathcal{S}$.

Remark. Let $M$ be a manifold, $S$ a closed subset of $M$, and $\mathcal{S}$ a Whitney stratification of $S$. Let $x$ and $x^{\prime}$ be two points in the same connected component of a stratum of $\mathcal{S}$. Then there exists a homeomorphism $h$ of $M$ onto itself which preserves $S$ and $\mathcal{S}$ such that $h(x)=x^{\prime}$. This follows from Thom's theory [4] and we will prove it 
below. In the case $\mathcal{S}$ has only two strata, it is quite easy to prove by an argument due to Thom [4, p. 242].

We sketch Thom's argument for the two strata case here. The only non-trivial case is when the two strata satisfy $X<Y$ and the two points $x$ and $x^{\prime}$ are in $X$. In this case $X$ is closed and $X \subset \bar{Y}=Y \cup X$.

For simplicity, we will suppose that $M$ is compact, though it is not difficult to modify the argument to make it work in the case $M$ is non-compact.

Let $N$ be a small tubular neighborhood of $X$ in $M$, let $\pi: N \rightarrow X$ be a smooth retraction, and let $\rho$ be a smooth function on $M$ such that $\rho \geq 0, X=\{\rho=0\}$, and at a point $x \in X, \rho$ is non-degenerate on the normal plane to $X$ in the sense that the Hessian matrix of $\rho$ at $x$ has rank equal to the codimension of $X$.

Now let $x$ and $x^{\prime}$ be two points in the same connected component of $X$. Let $v_{X}$ be a smooth vector field on $X$ such that the trajectory of $v$ starting at $x$ arrives at $x^{\prime}$ at time $t=1$.

For $\varepsilon>0$ sufficiently small, the subset $M_{\varepsilon}=\{\rho=\varepsilon\}$ of $N$ is compact, and $\pi: M_{\varepsilon} \rightarrow X$ is a submersion. Furthermore, $Y_{\varepsilon}=M_{\varepsilon} \cap Y$ is compact, and it follows from condition $b$ that $\pi: Y_{\varepsilon} \rightarrow X$ is a submersion for $\varepsilon$ sufficiently small. It follows easily that there is a vector field $v$ on $M-X$ and an $\varepsilon_{1}>0$ such that $v$ is tangent along $Y$, and the following hold.

$$
\begin{array}{ll}
v \rho(m)=0 & \text { if } m \in M-X \text { and } \rho(m)<\varepsilon_{1}, \\
\pi_{*} v(m)=v_{X}(\pi m) & \text { if } m \in M-X \text { and } \rho(m)<\varepsilon_{1} .
\end{array}
$$

From (5.1) and the compactness of $M$, it follows that the trajectory of $v$ starting at any point of $M-X$ is defined for all time. Hence $v$ generates a one-parameter group $\left\{h_{t}^{0}: t \in \mathbb{R}\right\}$ of diffeomorphisms of $M-X$. Clearly $v_{X}$ generates a oneparameter group $\left\{h_{t}^{X}: t \in \mathbb{R}\right\}$ of diffeomorphisms of $X$. Let $h_{t}: M \rightarrow M$ be defined by $h_{t} \mid M-X=h_{t}^{0}$ and $h_{t} \mid X=h_{t}^{X}$. It follows from (5.1) and (5.2) that $h_{t}^{X} \pi(m)=\pi h_{t}^{0}(m)$ if $m \in M-X$ and $\rho(m)<\varepsilon_{1}$. Hence $h_{t}$ is a homeomorphism of $M$. Clearly $h_{t}$ preserves $X$, and furthermore $h_{t}$ preserves $Y$, since $v$ is tangent along $Y$. Finally $h_{1}(x)=x^{\prime}$ since the trajectory of $v_{X}$ starting at $x$ arrives at $x^{\prime}$ at time $t=1$.

Thus $h=h_{1}$ is the required homeomorphism of $M$.

\section{Tubular NeIghborhoOdS}

In this section, we define the notion of a tubular neighborhood of a submanifold of a manifold, and prove an existence and uniqueness theorem for tubular neighborhoods. Our existence and uniqueness theorem is slightly more general than the standard one (cf., Lang [2]). The method of proof we use was suggested by A. Ogus.

We recall that a vector bundle $E$ over a smooth manifold $M$ is said to be smooth if the coordinate transition functions which define $E$ are smooth functions. By a smooth inner product on a vector bundle $E$, we will mean a rule which assigns to each fibre $E_{u}$ of $E$ an inner product $(\cdot, \cdot)_{u}$ on $E_{u}$ and which has the following property: if $U$ is any open set in $M$ and $s_{1}, s_{2}$ are two smooth sections of $E$ above $U$ then the mapping $u \rightarrow\left(s_{1}(u), s_{2}(u)\right)_{u}$ is smooth. From now on, we will assume all vector bundles and inner products on vector bundles are smooth, unless the contrary is explicitly stated. By a (smooth) inner product bundle, we mean a pair consisting of a (smooth) vector bundle $E$ and a (smooth) inner product on $E$. 
If $\pi: E \rightarrow M$ is an inner product bundle over a manifold, and $\epsilon$ is a positive function on $M$, then the open $\epsilon$-ball bundle $B_{\epsilon}$ of $E$ will be defined as the set of $e$ in $E$ such that $\|e\|<\epsilon(\pi e)$, where $\|e\|$ is defined as $(e, e)^{1 / 2}$.

Let $M$ be a manifold and $X$ a submanifold.

Definition. A tubular neighborhood $T$ of $X$ in $M$ is a triple $(E, \epsilon, \varphi)$, where $\pi: E \rightarrow$ $X$ is an inner product bundle, $\epsilon$ is a positive smooth function on $X$, and $\varphi$ is a diffeomorphism of $B_{\epsilon}$ onto an open subset of $M$ which commutes with the zero section $\zeta$ of $E$ :

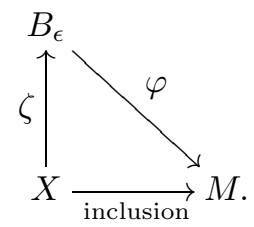

We set $|T|=\varphi\left(B_{\epsilon}\right)$. By the projection associated to $T$, we mean the mapping $\pi_{T}=\pi \circ \varphi^{-1}:|T| \rightarrow X$. By the tubular function associated to $T$, we mean the non-negative real valued function

$$
\rho_{T}=\rho \circ \varphi^{-1}:|T| \rightarrow \mathbb{R} \quad \text { where } \quad \rho(e)=\|e\|^{2} \quad \text { for all } \quad\|e\| \in|T| .
$$

It follows from these definitions that $\pi_{T}$ is a retraction of $|T|$ on $X$, i.e., the composition

$$
X \stackrel{\text { inclusion }}{\longrightarrow}|T| \stackrel{\pi_{T}}{\longrightarrow} X
$$

is the identity. Also, $X$ is the 0 -set of $\rho_{T}$, the differential of $\rho_{T}$ vanishes only on $X$, and (in the case $\mu \geq 2$ ) at a point $x \in X, \rho_{T}$ is non-degenerate on the normal plane to $X$ in the sense that the Hessian matrix of $\rho$ at $x$ has rank equal to the co-dimension of $X$.

If $U$ is a subset of $X$, the restriction $T \mid U$ of $T$ to $U$ is defined as $(E|U, \epsilon| U, \varphi \mid U)$.

If $T=(E, \epsilon, \varphi)$ and $T^{\prime}=\left(E^{\prime}, \epsilon^{\prime}, \varphi^{\prime}\right)$ are two tubular neighborhoods of $X$ in $M$, an inner product bundle isomorphism $\psi: E \rightarrow E^{\prime}$ will be said to be an isomorphism of $T$ with $T^{\prime}$ if there exists a positive continuous function $\epsilon^{\prime \prime}$ on $X$ such that $\epsilon^{\prime \prime} \leq$ $\min \left(\epsilon, \epsilon^{\prime}\right)$ and $\varphi^{\prime} \circ \psi\left|B_{\epsilon^{\prime \prime}}=\varphi\right| B_{\epsilon^{\prime \prime}}$. Note that if this holds, then $\pi_{T}\left|\varphi B_{\epsilon^{\prime \prime}}=\pi_{T^{\prime}}\right| \varphi B_{\epsilon^{\prime \prime}}$ and $\rho_{T}\left|\varphi B_{\epsilon^{\prime \prime}}=\rho_{T^{\prime}}\right| \varphi B_{\epsilon^{\prime \prime}}$. We say $T$ and $T^{\prime}$ are isomorphic and write $T \sim T^{\prime}$ if there exists an isomorphism from $T$ to $T^{\prime}$.

A smooth mapping $f: M \rightarrow P$ will be said to be a submersion if $d f: T M_{x} \rightarrow$ $T P_{f(x)}$ is onto for each $x \in M$.

Throughout the rest of this section, let $f: M \rightarrow P$ be a smooth mapping, and $X$ a submanifold of $M$.

A tubular neighborhood $T$ of $X$ in $M$ will be said to be compatible with $f$ if $f \circ \pi_{T}=f|| T \mid$. A mapping $h$ of $M$ into itself will be said to be compatible with $f$ if $f \circ h=f$. A homotopy $H: M \times I \rightarrow M$ of $M$ into itself will be said to be compatible with $f$ if $f \circ H_{t}=f$ for all $t \in I(=[0,1])$. By an isotopy of $M$, we will mean a smooth mapping $H: M \times I \rightarrow M$ such that $H_{0}=\mathrm{id}: M \rightarrow M$ and $H_{t}: M \rightarrow M$ is a diffeomorphism for all $t \in I$. If $h$ is a diffeomorphism of $M$ into itself, the support of $h$ will mean the closure of $\{x \in M: h(x) \neq x\}$. Likewise, if $H: M \times I \rightarrow M$ is an isotopy, the support of $H$ will mean the closure of $\{x \in M: \exists t \in I, H(x, t) \neq x\}$.

If $M^{\prime}$ is a second manifold, $X^{\prime}$ is a submanifold of $M^{\prime}$, and $h:(M, X) \rightarrow\left(M^{\prime}, X^{\prime}\right)$ is a diffeomorphism, then for any tubular neighborhood $T=(E, \epsilon, \varphi)$ of $X$ we define a tubular neighborhood $h_{*} T$ of $X^{\prime}$ by $h_{*} T=\left(\left(h^{-1}\right)^{*} E, \epsilon \circ h^{-1}, h \circ \varphi\right)$. 
We will begin by stating and proving a uniqueness theorem for tubular neighborhoods, and then we will derive an existence theorem from the uniqueness theorem. This procedure of deducing the existence theorem from the uniqueness theorem was suggested to us by A. Ogus.

The simplest uniqueness theorem for tubular neighborhoods states that if $\mathrm{X}$ is closed and $T_{0}$ and $T_{1}$ are tubular neighborhoods of $X$ in $M$, then there exists a diffeomorphism $h$ of $M$ onto itself which leaves $X$ point-wise fixed such that $h_{*} T_{0} \sim T_{1}$. Moreover, $h$ can be chosen so that there is an isotopy $H$ of $M$ with $H_{1}=h$ which leaves $X$ point-wise fixed. We can generalize this result in various ways.

First, under the hypothesis that $T_{0}$ and $T_{1}$ are compatible with $f$ and $f \mid X$ is a submersion, we can choose $h$ and $H$ to be compatible with $f$. Secondly, if $T_{0}\left|U \sim T_{1}\right| U$ for some open set $U$ in $X$, and $Z$ is a closed subset of $M$ such that $Z \cap X \subseteq U$, then we can choose $h$ and $H$ to leave $Z$ point-wise fixed.

The following proposition implies these statements, and has some other wrinkles as well. We will use it in its full generality.

Proposition 6.1 (Uniqueness of tubular neighborhoods). Suppose $\mu \geq 2$. Suppose the submanifold $X$ of $M$ is closed, and $f \mid X: X \rightarrow P$ is a submersion. Let $U$ be an open subset of $X$, let $U^{\prime}$ and $V^{\prime}$ be closed subsets of $X$, let $V$ be an open subset of $M$, and suppose $U^{\prime} \subseteq U$ and $V^{\prime} \subseteq V$. Let $T_{0}$ and $T_{1}$ be tubular neighborhoods of $X$ in $M$ which are compatible with $f$ and suppose there is an isomorphism $\psi_{0}: T_{0}\left|U \rightarrow T_{1}\right| U$. Then there is an isotopy $H: M \times I \rightarrow M$, compatible with $f$, leaving $X$ point-wise fixed, and with support in $V-U^{\prime}$, such that $h_{*} T_{0}\left|V^{\prime} \cup U^{\prime} \sim T_{1}\right| V^{\prime} \cup U^{\prime}$, where $h=H_{1}$. Moreover, if $N$ is any neighborhood of the diagonal in $M \times M$, we can choose $H$ such that $\left(H_{t}(x), x\right) \in N$ for any $t \in I$ and $x \in M$. Also, we can choose $H$ so that there is an isomorphism $\psi: h_{*} T_{0}\left|V^{\prime} \cup U^{\prime} \sim T_{1}\right| V^{\prime} \cup U^{\prime}$ such that $\psi\left|U^{\prime}=\psi_{0}\right| U^{\prime}$.

Proof. Let $m=\operatorname{dim} M, c=\operatorname{cod} X$, and $p=\operatorname{dim} P$. For $k<m$, let $\mathbb{R}^{k}$ be embedded as $\mathbb{R}^{k} \times 0_{m-k}$ in $\mathbb{R}^{m}$. We will say that we are in the local case when $V^{\prime}$ is compact and there exists a diffeomorphism $\Phi$ of $M$ onto an open subset of $\mathbb{R}^{m}$, such that $\Phi(X)=\mathbb{R}^{m-c} \cap \Phi(M)$, and a diffeomorphism $\Psi$ of $P$ onto an open subset of $\mathbb{R}^{p}$ such that the following diagram commutes, where $\pi$ is given by $\pi\left(x_{1}, \cdots, x_{m}\right)=\left(x_{1}, \cdots, x_{p}\right)$ :

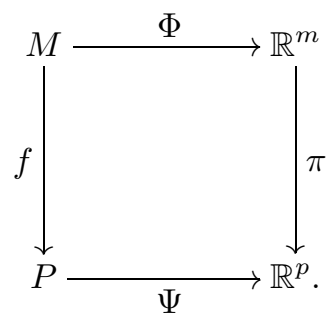

There are two steps in the proof:

Step 1. Reduction to the local case. From the hypothesis that $f \mid X$ is a submersion, it follows that for each $x \in X$ there exists an open neighborhood $W_{x}$ of $x$ in $M$, a diffeomorphism $\Phi_{x}$ of $W_{x}$ onto an open subset of $\mathbb{R}^{m}$ such that $\Phi\left(W_{x} \cap X\right)=\Phi\left(W_{x}\right) \cap \mathbb{R}^{m-c}$, and a diffeomorphism $\Psi_{x}$ of $f\left(W_{x}\right)$ onto an open 
subset of $\mathbb{R}^{p}$ such that the following diagram commutes:

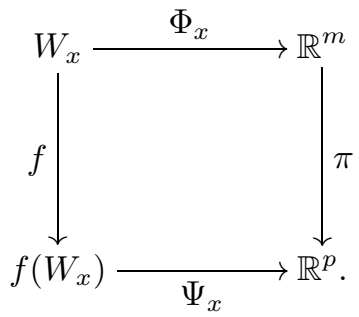

Furthermore, we may suppose each $W_{x}$ is relatively compact, and that

$$
\begin{aligned}
& W_{x} \cap V^{\prime} \neq \varnothing \Longrightarrow W_{x} \subseteq V \\
& W_{x} \cap U^{\prime} \neq \varnothing \Longrightarrow W_{x} \cap X \subseteq U .
\end{aligned}
$$

Then $\{M-X\} \cup\left\{W_{x}\right\}$ is a cover of $\mathrm{M}$, so that there exists a locally finite refinement of it, which we may take to be of the form $\{M-X\} \cup\left\{W_{i}\right\}$, where each $W_{i}$ is open in $M$ and is contained in $W_{x_{i}}$ for some $x_{i} \in X$. Since $M$ has a countable basis for its topology, the collection $\left\{W_{i}\right\}$ is countable. Now we discard all $W_{i}$ for which $W_{i} \cap U^{\prime} \neq \varnothing$ or $W_{i} \cap V^{\prime}=\varnothing$, and we index the remaining $W_{i}$ 's by the positive integers. Then we have $V^{\prime} \subseteq U \cup W_{1} \cup W_{2} \cup \cdots$, and $W_{i} \subseteq V$ for all $i$, by (6.1).

We can choose closed sets $W_{i}^{\prime} \subseteq W_{i} \cap X$ such that $V^{\prime} \subseteq U \cup W_{1}^{\prime} \cup W_{2}^{\prime} \cup \cdots$. Since $W_{i}^{\prime} \subseteq W_{x_{i}}$, and the latter is relatively compact, it follows that $W_{i}^{\prime}$ is compact.

Now we construct by induction a sequence $H^{0}, H^{1}, H^{2}, \cdots$ of isotopies of $M$ into itself and a sequence $\psi_{0}, \psi_{1}, \psi_{2}, \cdots$ of isomorphisms of tubular neighborhoods. We let $H^{0}$ be defined by $H_{t}^{0}=$ identity, $0 \leq t \leq 1$, and let $\psi_{0}$ be as given in the statement of the proposition.

For the inductive step, we suppose that $H^{0}, H^{1}, \cdots, H^{i-1}$ and $\psi_{0}, \psi_{1}, \cdots, \psi_{i-1}$ have been constructed, are compatible with $f$, and leave $X$ point-wise fixed. We let $G^{j}$ be the isotopy of $M$ defined by $G_{t}^{j}=H_{t}^{j} \circ H_{t}^{j-1} \circ \cdots \circ H_{t}^{0}$. We set $g^{j}=G_{1}^{j}$. We let $U_{j}=U \cup W_{1} \cup W_{2} \cup \cdots \cup W_{j}$ and suppose supp $G^{i-1} \subseteq U_{i-1} \cap V$. Furthermore, we suppose $\left(G_{t}^{i-1}(x), x\right) \in N$ for all $x \in M$ and $t \in[0,1]$, and that $\psi_{i-1}$ is an isomorphism of tubular neighborhoods $g_{*}^{i-1} T_{0}\left|\bar{U}_{i-1}^{*} \rightarrow T_{1}\right| \bar{U}_{i-1}^{*}$, where $U_{i-1}^{*}$ is an open neighborhood of $U^{\prime} \cup W_{1}^{\prime} \cup \cdots \cup W_{i-1}^{\prime}$ in $X$.

Then it follows from the local case of the proposition that $H^{i}$ and $\psi_{i}$ can be chosen so that the conditions of the induction are satisfied. For, let $W_{i}^{0}$ be an open subset of $W_{i}$ such that $W_{i}^{\prime} \subseteq W_{i}^{0}$ and $W_{i}^{0}$ is relatively compact in $W_{i}$, and let $U_{i}^{*}$ be an open neighborhood of $U^{\prime} \cup W_{1}^{\prime} \cup \cdots \cup W_{i}^{\prime}$ in $X$ whose closure lies in $U_{i-1}^{*} \cup W_{i}^{0}$. From the local case, it follows that we can construct an isotopy $H^{i}$ of $W_{i}$, compatible with $f$, leaving $X \cap W_{i}$ point-wise fixed, and with support in $W_{i}^{0}$ such that $h_{*}^{i} g_{*}^{i-1} T_{0}\left|\bar{U}_{i}^{*} \cap W_{i} \sim T_{1}\right| \bar{U}_{i}^{*} \cap W_{i}$, where $h^{i}=H_{1}^{i}$. (This is because $g_{*}^{i-1} T_{0}\left|\bar{U}_{i-1}^{*} \cap W_{i} \sim T_{1}\right| \bar{U}_{i-1}^{*} \cap W_{i}$ and $\bar{U}_{i}^{*} \subset U_{i-1}^{*} \cup W_{i}^{0}$.) Moreover, we may choose $H^{i}$ so that $H_{t}^{i}$ is arbitrarily close to the identity for all $t$, and so there is an isomorphism

$$
\psi_{i}: h_{*}^{i} g_{*}^{i-1} T_{0}\left|\bar{U}_{i}^{*} \cap W_{i} \rightarrow T_{1}\right| \bar{U}_{i}^{*} \cap W_{i}
$$

such that

$$
\psi_{i}\left|\bar{U}_{i}^{*} \cap W_{i} \cap \bar{U}_{i-1}^{*}=\psi_{i-1}\right| \bar{U}_{i}^{*} \cap W_{i} \cap \bar{U}_{i-1}^{*}
$$


Since supp $H^{i}$ is a compact subset of $W_{i}$, we may extend $H^{i}$ to an isotopy of $M$ whose support lies in $W_{i}$. Likewise, we may extend $\psi_{i}$ to all of $U_{i}^{*}$ by letting $\psi_{i}\left|U_{i-1}^{*}=\psi_{i-1}\right| U_{i-1}^{*}$. Then $H^{i}$ and $\psi_{i}$ satisfy the conditions of the induction.

Now if it is true that the sequence $\left\{G_{t}^{j}(x)\right\}$ is eventually constant in a neighborhood of any point $x \in M$, we can set

$$
H_{t}(x)=\lim _{i \rightarrow \infty} G_{t}^{i}(x)
$$

and

$$
\psi(x)=\lim _{i \rightarrow \infty} \psi_{i}(x)
$$

(since the latter is eventually constant in a neighborhood of any point). If we choose $N$ so that the projection $\pi_{2}: \bar{N} \rightarrow M$ is proper (where $\pi_{2}$ denotes the projection on the second factor), then it is easily seen that the sequence $G_{t}^{i}(x)$ is eventually constant in a neighborhood of any point $x \in M$, and that $H$ and $\psi$ have the required properties.

This completes the reduction to the local case.

Step 2. Proof in the local case. Let $T_{0}=\left(E_{0}, \epsilon_{0}, \varphi_{0}\right)$ and $T_{1}=\left(E_{1}, \epsilon_{1}, \varphi_{1}\right)$. We will first construct an isomorphism $\psi: E_{0} \rightarrow E_{1}$ of inner product bundles which extends $\psi_{0} \mid U^{\prime}$, and then construct the isotopy $H$ to have the required properties.

The tubular neighborhood $T_{i}(i=0,1)$ gives a natural identification $\alpha_{i}$ of $E_{i}$ with the normal bundle $\nu_{X}$ of $X$ in $M$. Explicitly, if $x \in X$, the restriction of $\alpha_{i}$ to the fibre $E_{i, x}$ is the composition

$$
E_{i, x}=T\left(E_{i, x}\right)_{0} \stackrel{d \varphi_{i}}{\longrightarrow} T M_{x} \stackrel{\text { projection }}{\longrightarrow} T M_{x} / T X_{x}=\nu_{X, x} .
$$

Let $\beta=\alpha_{1}^{-1} \alpha_{0}: E_{0} \rightarrow E_{1}$. We may consider $\beta$ as a section of $\operatorname{Iso}\left(E_{0}, E_{1}\right)$, where the latter is the bundle whose fibre over $x$ is the space of isomorphism of $E_{0, x}$ into $E_{1, x}$. In general $\beta$ will not be of class $C^{\mu}$, only of class $C^{\mu-1}$; however, we may approximate $\beta$ arbitrarily closely (in the $C^{\mu-1}$ topology) on any compact subset of $X$ by a section $\beta_{1}$ of class $C^{\mu}$.

To construct $\psi$, we will need the following well-known lemma in linear algebra.

Lemma. Let $V$ and $W$ be vector spaces, provided with inner products $k$ and $\ell$. Let $L: V \rightarrow W$ be a vector space isomorphism. Then there exists a unique positive definite self-adjoint linear mapping $H: W \rightarrow W$ such that $H \circ L: V \rightarrow W$ preserves inner products.

Remark 1. It is easily seen that this is equivalent to the assertion that any invertible matrix $L$ of real numbers has a unique decomposition $L=H^{-1} U$ where $H$ is a positive definite symmetric matrix and $U$ is an orthogonal matrix.

Remark 2. Similarly, it is easily verified that there exists a unique positive definite self-adjoint linear mapping $H_{1}: V \rightarrow V$ such that $L \circ H_{1}: V \rightarrow W$ preserves inner products, and that $H_{1}=L^{-1} H L$.

Proof of the lemma. Existence. Let $e_{1}, \cdots, e_{n}$ be an orthonormal basis for $\mathrm{V}$, and let $A=\left(\alpha_{i j}\right)$ be the matrix given by $\alpha_{i j}=\left(L e_{i}, L e_{j}\right)_{\ell}$. Then $\alpha_{i j}$ is symmetric and positive definite. It follows from the spectral theorem for symmetric positive definite matrices that we may choose the basis $e_{1}, \cdots e_{n}$ so that $\left(\alpha_{i j}\right)$ is a diagonal matrix: $\alpha_{i j}=\lambda_{i} \delta_{i j}$ (where $\delta_{i j}$ is the Kronecker delta symbol). Let $f_{i}=L\left(e_{i}\right) / \sqrt{\lambda_{i}}$. 
Then $f_{1}, \cdots, f_{n}$ is an orthonormal basis of $W$. Let $H: W \rightarrow W$ be given by $H\left(f_{i}\right)=f_{i} / \sqrt{\lambda_{i}}$. Then $H$ has the required properties.

Uniqueness. If there were two, $H$ and $H^{\prime}$, we would have that $U=(H L) \circ$ $\left(H^{\prime} L\right)^{-1}$ is orthogonal. Then $U H^{\prime} L=H L$ so $U H^{\prime}=H$. Taking adjoints, we then obtain $H^{\prime} U^{-1}=H$ so that $H^{\prime 2}=H^{\prime} U^{-1} U H^{\prime}=H^{2}$. This implies $H^{\prime}=H$, since a positive definite self-adjoint mapping has only one positive definite self-adjoint square root.

Now we return to the proof of the uniqueness of tubular neighborhoods. For each $x \in X$, let $\eta_{x}$ be the unique self-adjoint positive definite linear automorphism of $E_{1, x}$ such that $\psi_{x}=\eta_{x} \circ \beta_{1, x}: E_{0, x} \rightarrow E_{1, x}$ preserves inner products. Clearly, $\psi=\left\{\psi_{x}\right\}$ is a smooth isomorphism of $E_{0}$ onto $E_{1}$, and it preserves inner products. From the fact that $\eta_{x}$ is positive definite and self-adjoint it follows that $(1-t) \mathrm{id}+t \eta_{x}$ is an automorphism of $E_{1, x}$ for $0 \leq t \leq 1$. Hence if $\beta_{1}$ is chosen sufficiently $C^{1}$-close to $\beta$, it follows that

$$
(1-t) \beta_{1}+t \psi: E_{0} \rightarrow E_{1}
$$

is an isomorphism for $0 \leq t \leq 1$. Moreover, if we choose $\beta_{1}$ so that $\beta_{1}=\beta$ in a neighborhood of $U^{\prime}$ (which we may do since $\beta \mid U=\psi_{0}$ by definition of $\beta$ ), then $\eta=$ identity in a neighborhood of $U^{\prime}$, so that $\psi\left|U^{\prime}=\psi_{0}\right| U^{\prime}$.

Since we are in the local case, we may suppose without loss of generality that $M$ is open in $\mathbb{R}^{m}, P$ is open in $\mathbb{R}^{p}, X=\mathbb{R}^{m-c} \cap M$, and $f=\pi \mid M$. It is easily seen that there exists a neighborhood $V_{1}$ of $V^{\prime}$ in $V$ such that for all $m \in V_{1}$, we have that

$$
g_{t}(m)=\varphi_{1} \circ\left\{(1-t) \beta_{1}+t \psi\right\} \circ \varphi_{0}^{-1}(m)
$$

is defined. Since $V^{\prime} \subseteq X$, we have $g_{t} \mid V^{\prime}=$ inclusion. Since $V^{\prime}$ is compact there exists an open neighborhood $V_{2}$ of $V^{\prime}$ in $V_{1}$ such that $V_{2} \subseteq g_{t}\left(V_{1}\right)$ for $0 \leq t \leq 1$. Let $\rho$ be a $C^{\infty}$ function on $M$ which is identically 1 in a neighborhood of $V^{\prime}$ and which has compact support $\subseteq V_{2}$. Let $G_{s, t}: M \rightarrow M$ be defined by

$$
G_{s, t}(m)=\left\{\begin{array}{lll}
\left((1-\rho(m)) m+\rho(m) g_{t} g_{s}^{-1}(m)\right. & \text { if } & m \in V_{2}, \\
m & \text { if } & m \in M-V_{2} .
\end{array}\right.
$$

Then $G_{s, t}$ is a smooth mapping for $0 \leq s, t \leq 1$, and it depends smoothly on $s$ and $t$. Since $G_{t, t}=$ identity and there is a compact set which contains the support of $G_{s, t}$ for all $s$ and $t$, it follows that there exists $\delta>0$ such that $G_{s, t}$ is a diffeomorphism for $|s-t|<\delta$. Let $n$ be a positive integer such that $1 / n<\delta$ and set

$$
\widetilde{H}_{t}=G_{0, \frac{t}{n}} \circ G_{\frac{t}{n}, \frac{2 t}{n}} \circ \cdots \circ G_{\frac{(n-1) t}{n}, t} .
$$

Then $\widetilde{H}_{t}$ is an isotopy of $M$, and it follows from the definition of $\widetilde{H}$ that $\widetilde{H}_{1}=g_{1} \circ g_{0}^{-1}$ in a sufficiently small neighborhood of $V^{\prime}$. Also it follows from the definitions that $g_{t}$ and $\widetilde{H}_{t}$ are the identity in a sufficiently small neighborhood of $U^{\prime}$ for all t. Thus $\widetilde{H}_{1}=g_{1} \circ g_{0}^{-1}$ in a sufficiently small neighborhood of $U^{\prime} \cup V^{\prime}$. Clearly $\operatorname{supp} \widetilde{H} \subseteq V_{2} \subseteq V$.

Furthermore, $\widetilde{H}_{1} \circ g_{0} \circ \varphi_{0}=g_{1} \circ \varphi_{0}=\varphi_{1} \circ \psi$ in a small neighborhood of $U^{\prime} \cup V^{\prime}$. Thus $\psi$ is an isomorphism of $\left(\widetilde{H}_{1} g_{0}\right)_{*} T_{0} \mid U^{\prime} \cup V^{\prime}$ with $T_{1} \mid U^{\prime} \cup V^{\prime}$.

It is clear from the construction that $\widetilde{H}$ is compatible with $f$ and leaves $X$ pointwise fixed. By choosing the function $\rho$ used in the construction of $G$ to have support 
in a very small neighborhood of $V^{\prime}$, we may arrange for $\widetilde{H}_{t}$ to be as close to the identity (in the compact-open topology) as we like.

It is easily seen that there exists an isotopy $\widehat{H}$ of $M$ which is compatible with $f$ and leaves $X$ point-wise fixed, such that $\widehat{H}_{t}$ is the identity in a neighborhood of $U^{\prime}$ and $\widehat{H}_{1}=g_{0}$ in a neighborhood of $V^{\prime}$. Let $H_{t}=\widetilde{H}_{t} \circ \widehat{H}_{1}$. Then $H$ is an isotopy of $M$ with all the required properties.

Now we state and prove the existence theorem for tubular neighborhoods.

Proposition 6.2. Suppose $f \mid X: X \rightarrow P$ is a submersion. Let $U$ be an open subset of $X$ and let $T_{0}$ be a tubular neighborhood of $U$ in $X$. Let $U^{\prime}$ be a subset of $U$ which is closed in $X$. Then there exists a tubular neighborhood $T$ of $X$ in $M$ such that $T\left|U^{\prime} \sim T_{0}\right| U^{\prime}$

Proof. It is enough to consider the case when $X$ is closed in $M$. For, in general, there is an open subset $M_{0}$ in $M$ such that $X$ is a closed subset of $M_{0}$, since $X$ is locally closed in $M$. Clearly a tubular neighborhood of $X$ in $M_{0}$ is a tubular neighborhood of $X$ in $M$.

The local case of this proposition is trivial.

To prove the proposition in general, we take a locally finite family $\left\{W_{i}\right\}$ of open sets in $M$ having the following properties:

(a) For each $i$, there is a coordinate chart $\varphi_{i}: W_{i} \rightarrow \mathbb{R}^{n}$ such that $\varphi_{i}\left(W_{i} \cap X\right)=$ $\varphi_{i}\left(W_{i}\right) \cap \mathbb{R}^{n-c}$ (where $c=\operatorname{cod} X$ ) and such that there is a coordinate chart $\psi_{i}: f\left(W_{i}\right) \rightarrow \mathbb{R}^{p}$ such that the following diagram commutes

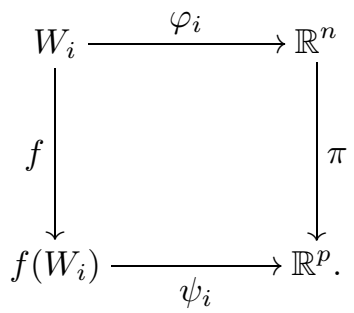

(b) each $\bar{W}_{i}$ is compact, and

(c) $\left\{W_{i} \cap X\right\}$ is a cover of $X$.

Furthermore, we can choose closed sets $W_{i}^{\prime}$ of $X$ such that $W_{i}^{\prime} \subseteq W_{i}$ and $\left\{W_{i}^{\prime}\right\}$ is a cover of $X$. Since $M$ has a countable basis for its topology, the family $\left\{W_{i}\right\}$ is countable. We will suppose that it is indexed by the positive integers. For each positive integer $i$ we let $U_{i}=U \cup W_{1} \cup \cdots \cup W_{i}$ and $U_{i}^{\prime}=U^{\prime} \cup W_{1}^{\prime} \cup \cdots \cup W_{i}^{\prime}$. We let $U_{0}=U$ and $U_{0}^{\prime}=U^{\prime}$.

Now we construct by induction on $i$ an open neighborhood $U_{i}^{\prime \prime}$ of $U_{i}^{\prime}$ in $X$ and a tubular neighborhood $T_{i}$ of $U_{i}^{\prime \prime}$. We take $T_{0}$ as given. For the inductive step, we suppose $U_{i-1}^{\prime \prime}$ and $T_{i-1}$ have been constructed. We let $U_{i}^{\prime \prime}$ be any open neighborhood of $U_{i}^{\prime}$ in $X$ which is relatively compact in $W_{i} \cup U_{i-1}^{\prime \prime}$.

Since $\bar{U}_{i}^{\prime \prime} \subseteq W_{i} \cup U_{i-1}^{\prime \prime}$, there exist open sets $A$ and $B$ in $U_{i}^{\prime \prime}$ such that $U_{i}^{\prime \prime}=$ $A \cup B, \bar{A} \subseteq W_{i}-U_{i-1}^{\prime}$ and $\bar{B} \subseteq U_{i-1}^{\prime \prime}$. Since the existence theorem for tubular neighborhoods is true in the local case, we may choose a tubular neighborhood $T_{i}^{\prime}$ of $W_{i} \cap X$ in $W_{i}$. Then we have two tubular neighborhoods of $U_{i-1}^{\prime \prime} \cap W_{i} \cap X$ in $M$, namely the restrictions of $T_{i}^{\prime}$ and $T_{i-1}$. Since $A \cap B$ is relatively compact in $\left(U_{i}^{\prime \prime}-U_{i-1}^{\prime}\right) \cap W_{i} \cap X$, we may find a diffeomorphism $h$ of $M$ onto itself leaving 
$X$ point-wise fixed such that $h_{*} T_{i-1}\left|A \cap B \sim T_{i}^{\prime}\right| A \cap B$. Furthermore, we may suppose $h$ is compatible with $f$ and $h$ is the identity outside an arbitrarily small neighborhood of $\overline{A \cap B}$; in particular, that $h$ is the identity in a neighborhood of $U_{i-1}^{\prime}$. Since $h_{*} T_{i-1}\left|A \cap B \sim T_{i}^{\prime}\right| A \cap B$ there is a tubular neighborhood $T_{i}$ of $U_{i}^{\prime \prime}=A \cup B$ in $M$ such that $T_{i}\left|A \sim T_{i}^{\prime}\right| A$ and $T_{i}\left|B \sim h_{*} T_{i-1}\right| B$. Clearly $T_{i}$ is compatible with $f$.

Furthermore, $T_{i} \sim T_{i-1}$ in a neighborhood of $U_{i-1}^{\prime}$. It follows easily that there is a tubular neighborhood $T$ of $X$ in $M$ such that $T \sim T_{i}$ in a neighborhood of $U_{i}^{\prime}$ for all $i$, and that this tubular neighborhood is compatible with $f$.

\section{Control Data}

Throughout this section, let $M$ be a manifold and $\mathcal{S}$ a Whitney stratification of a subset $S$ of $M$.

Suppose that for each stratum $X$ of $\mathcal{S}$ we are given a tubular neighborhood $T_{X}$ of $X$ in $M$. Let $\pi_{X}:\left|T_{X}\right| \rightarrow X$ denote the projection associated to $T_{X}$ and $\rho_{X}:\left|T_{X}\right| \rightarrow \mathbb{R}$ be the tubular function associated to $T_{X}$.

Definition. The family $\left\{T_{X}\right\}$ of tubular neighborhoods will be called control data for $\mathcal{S}$ if the following commutation relations are satisfied: if $X$ and $Y$ are strata and $X<Y$, then

$$
\begin{aligned}
& \pi_{X} \pi_{Y}(m)=\pi_{X}(m), \\
& \rho_{X} \pi_{Y}(m)=\rho_{X}(m)
\end{aligned}
$$

for all $m$ such that both sides of the equation are defined, i.e., all $m \in\left|T_{X}\right| \cap\left|T_{Y}\right|$ such that $\pi_{Y}(m) \in\left|T_{X}\right|$.

If $f$ maps $M$ into $P$, then the family $\left\{T_{X}\right\}$ will be said to be compatible with $f$ if for all $X \in \mathcal{S}$ and all $m \in\left|T_{X}\right|$, we have $f \pi_{X}(m)=f(m)$.

Proposition 7.1. If $f: M \rightarrow P$ is smooth and $f \mid X$ is a submersion into $P$ for each stratum $X$, then there exists a family $\left\{T_{X}\right\}$ of control data for $\mathcal{S}$ which is compatible with $f$.

For the proof of the proposition, we will need Lemma 7.3 below. The proof of Lemma 7.3 depends on Lemma 7.2. which says (roughly speaking) that every tubular neighborhood is locally like a standard example.

Definition. By the standard tubular neighborhood $T_{m, c}$ of $\mathbb{R}^{m-c} \times 0_{c}$ in $\mathbb{R}^{m}$, we mean the triple $(E, \epsilon, \varphi)$, where $E$ is the trivial bundle over $\mathbb{R}^{m-c}$ with fibre $\mathbb{R}^{c}$ (provided with its standard inner product), $\epsilon=1$, and $\varphi: B_{\epsilon} \rightarrow \mathbb{R}^{m}$ is the restriction map of the identification mapping $\mathbb{R}^{m-c} \times \mathbb{R}^{c} \rightarrow \mathbb{R}^{m}$.

More generally if $U$ is open in $\mathbb{R}^{m-c}$, the standard tubular neighborhood of $U$ in $\mathbb{R}^{m}$ will mean $T_{m, c} \mid U$.

Lemma 7.2. If $X$ is a submanifold of $M, T_{X}$ is a tubular neighborhood of $X$, and $x \in X$, then there exists a coordinate chart $\varphi: U \rightarrow \mathbb{R}^{m}$, where $U$ is open in $M$ and $x \in U$, such that $\varphi(X \cap U)=\varphi(U) \cap \mathbb{R}^{m-c}$ (where $\left.c=\operatorname{cod} X\right)$ and such that

$$
\varphi_{*}\left(T_{X} \mid X \cap U\right) \sim T_{m, c} \mid \varphi(X \cap U) .
$$

Proof. Immediate from the definitions. 
If $T=(E, \epsilon, \varphi)$ is a tubular neighborhood of $X$ in $M$ and $\epsilon^{\prime}$ is any smooth positive function on $X$, we let $|T|_{\epsilon^{\prime}}=\varphi\left(B_{\epsilon} \cap \overline{B_{\epsilon^{\prime}}}\right),|T|_{\epsilon^{\prime}}^{0}=\varphi\left(B_{\epsilon} \cap B_{\epsilon^{\prime}}\right)$, and $\partial|T|_{\epsilon^{\prime}}=$ $\varphi\left(B_{\epsilon} \cap S_{\epsilon^{\prime}}\right)$ where $S_{\epsilon^{\prime}}$ is the $\epsilon^{\prime}$-sphere bundle in $E$, i.e., $S_{\epsilon^{\prime}}=\left\{v \in E:\|v\|=\epsilon^{\prime}(\pi(v))\right\}$ where $\pi: E \rightarrow X$ denotes the projection. Clearly $|T|_{\epsilon^{\prime}}$ is a smooth manifold with boundary $\partial|T|_{\epsilon^{\prime}}$, and interior $|T|_{\epsilon^{\prime}}^{0}$. We will say $\epsilon^{\prime}$ is admissible if $\epsilon^{\prime}<\epsilon$. In this case the projection $\pi_{T}:|T|_{\epsilon^{\prime}} \rightarrow X$ is a proper mapping.

Lemma 7.3. Let $X$ and $Y$ be disjoint submanifolds of $M$ such that the pair $(Y, X)$ satisfies condition $b$. Let $T$ be tubular neighborhood of $X$ in $M$. Then there exists a positive smooth function $\epsilon^{\prime}$ on $X$ such that the mapping

$$
\left(\rho_{T}, \pi_{T}\right): Y \cap|T|_{\epsilon^{\prime}}^{0} \rightarrow \mathbb{R} \times X
$$

is a submersion.

Proof. Let $\Sigma$ be the set of $y \in|T|$ such that the rank of the mapping

$$
\left(\rho_{T}, \pi_{T}\right): Y \cap|T| \rightarrow \mathbb{R} \times X
$$

at $y$ is $<\operatorname{dim}(\mathbb{R} \times X)$. The lemma is equivalent to the assertion that for any $x \in X$ there exists a neighborhood $N$ of $x$ in $M$ such that $N \cap \Sigma=\varnothing$. Since this is a purely local statement, it follows from Lemma 7.2 that it is enough to prove the proposition when $M=\mathbb{R}^{m}, X=\mathbb{R}^{m-c} \times 0_{c}$, and $T$ is the standard tubular neighborhood $T_{m, c}$ of $\mathbb{R}^{m-c}$ in $\mathbb{R}^{m}$. In this case $\pi_{T}$ is the orthogonal projection of $\mathbb{R}^{m}$ on $\mathbb{R}^{m-c}$, and $\rho_{T}$ is the function which is given by $\rho(y)=\operatorname{dist} .\left(y, \mathbb{R}^{m-c}\right)^{2}$.

Let $y \in|T|-\mathbb{R}^{m-c}$. The kernel of the differential of $\left(\pi_{T}, \rho_{T}\right)$ at $y$ is the orthogonal complement of $\left(\mathbb{R}^{m-c} \times 0_{c}\right) \oplus \widehat{y \pi_{T}(y)}$ in $\mathbb{R}^{m}$. The hypothesis that condition $b$ is satisfied implies that for $y$ near $\mathbb{R}^{m-c},\left(\mathbb{R}^{m-c} \times 0_{c}\right) \oplus \widehat{y \pi_{T}(y)}$ is close in the Grassmannian of $m-c+1$ planes in $m$ space to an $m-c+1$ plane which lies in $T Y_{y}$. Hence for $y$ near enough to $\mathbb{R}^{m-c}$, we have that $T Y_{y}$ is transversal to the kernel of the differential of $\left(\pi_{T}, \rho_{T}\right)$ at $y$, so that $\left(\pi_{T}, \rho_{T}\right) \mid Y$ is a submersion at $y$, i.e., $y \notin \Sigma$.

Proof of Proposition 7.1. Let $\mathcal{S}_{k}$ denote the family of strata of $\mathcal{S}$ of dimension $\leq k$, and let $S_{k}$ denote the union of all strata in $\mathcal{S}_{k}$. We will show by induction on $k$ that the proposition is true for $\mathcal{S}_{k}$ and $S_{k}$ in place of $\mathcal{S}$ and $S$.

For the inductive step, we suppose that for each stratum $X$ of dimension $<k$, we are given a tubular neighborhood $T_{X}$ of $X$, and this family of tubular neighborhoods satisfies the commutation relations.

By shrinking the $T_{X}$ if necessary, we may suppose that if $X$ and $Y$ are strata of dimension $<k$ which are not comparable (i.e., neither $Y<X$ nor $X<Y$ holds), then $\left|T_{X}\right| \cap\left|T_{Y}\right|=\varnothing$. To construct the $T_{X}$ on the strata of dimension $k$, we may do it one stratum at a time, since there are no commutation relations to be satisfied among the strata of the same dimension. Let $X$ be a stratum of dimension $k$.

We construct the tubular neighborhoods $T_{X}$ in two steps, as follows. For each $\ell \leq k$, we let $U_{\ell}$ denote the union of all $\left|T_{Y}\right|$ for $Y<X$ and $\operatorname{dim} Y \geq \ell$. We let $X_{\ell}=U_{\ell} \cap X$. In the first step, we construct a tubular neighborhood $T_{\ell}$ of $X_{\ell}$ by decreasing induction on $\ell$. In the inductive step, we will shrink various $\left|T_{Y}\right|$, but this is permitted, since we do it only a finite number of times. Then in the second step, we extend $T_{0}$ to a tubular neighborhood $T_{X}$ of $X$.

First step. For $\ell=k$, we have $X_{k}=\varnothing$, so there is nothing to construct. For the inductive step, we suppose that $T_{\ell+1}$ has been constructed and that the following 
special cases of the commutation relations are satisfied: if $Y<X, \operatorname{dim} Y \geq \ell+1$, $m \in\left|T_{\ell+1}\right| \cap\left|T_{Y}\right|$ and $\pi_{\ell+1}(m) \in\left|T_{Y}\right|$, where $\pi_{\ell+1}=\pi_{T_{\ell+1}}$, then

$\left(*_{\ell+1}\right)$

$$
\begin{aligned}
& \rho_{Y} \pi_{\ell+1}(m)=\rho_{Y}(m), \\
& \pi_{Y} \pi_{\ell+1}(m)=\pi_{Y}(m) .
\end{aligned}
$$

By replacing $T_{\ell+1}$ with a smaller tubular neighborhood if necessary, we may suppose that for $m \in\left|T_{\ell+1}\right|$ there is $Z<X$ with $\operatorname{dim} Z>\ell$ such that $m \in\left|T_{Z}\right|$ and $\pi_{\ell+1}(m) \in\left|T_{Z}\right|$.

To construct $T_{\ell}$ it is enough to construct $T_{\ell}$ on $\left|T_{Y}\right| \cap X$ for each stratum $Y<X$ of dimension $\ell$ separately, since if $Y$ and $Y^{\prime}$ are two strata of dimension $\ell$, we have $\left|T_{Y}\right| \cap\left|T_{Y^{\prime}}\right|=\varnothing$, since $Y$ and $Y^{\prime}$ are not comparable.

Thus, we wish to construct a tubular neighborhood $T_{X, Y}$ of $\left|T_{Y}\right| \cap X$ whose restriction to $\left|T_{Y}\right| \cap X_{\ell+1}$ is isomorphic to the restriction of $T_{\ell+1}$, such that the following commutation relation is satisfied: if $m \in\left|T_{X, Y}\right| \cap\left|T_{Y}\right|$ and $\pi_{X, Y}(m) \in$ $\left|T_{Y}\right|$, where $\pi_{X, Y}=\pi_{T_{X, Y}}$, then

$$
\begin{aligned}
& \rho_{Y} \pi_{X, Y}(m)=\rho_{Y}(m), \\
& \pi_{Y} \pi_{X, Y}(m)=\pi_{Y}(m) .
\end{aligned}
$$

By shrinking $\left|T_{Y}\right|$ if necessary, we may arrange that if $m \in\left|T_{\ell+1}\right| \cap\left|T_{Y}\right|$ and $\pi_{\ell+1}(m) \in\left|T_{Y}\right|$, then this commutation relation is already satisfied (with $\pi_{\ell+1}$ in place of $\left.\pi_{X, Y}\right)$ for the following reason. Since $m \in\left|T_{\ell+1}\right|$, there exists $Z<X$ with $\operatorname{dim} Z>\ell, m \in\left|T_{Z}\right|$ and $\pi_{\ell+1}(m) \in\left|T_{Z}\right|$. Since $\pi_{\ell+1}(m) \in\left|T_{Y}\right| \cap\left|T_{Z}\right|$, the last named space is not empty; hence $Y$ and $Z$ are comparable, and by dimension restrictions $Y<Z$. Therefore

$$
\begin{aligned}
& \rho_{Y} \pi_{\ell+1}(m)=\rho_{Y} \pi_{Z} \pi_{\ell+1}(m)=\rho_{Y} \pi_{Z}(m)=\rho_{Y}(m), \\
& \pi_{Y} \pi_{\ell+1}(m)=\pi_{Y} \pi_{Z} \pi_{\ell+1}(m)=\pi_{Y} \pi_{Z}(m)=\pi_{Y}(m) .
\end{aligned}
$$

(We may have to shrink $\left|T_{Y}\right|$ to guarantee that these equalities hold for all $m \in$ $\left.\left|T_{\ell+1}\right| \cap\left|T_{Y}\right|.\right)$

Furthermore, by shrinking $T_{Y}$ further if necessary, we may suppose that

$$
\left(\rho_{Y}, \pi_{Y}\right):\left|T_{Y}\right| \cap X \rightarrow \mathbb{R} \times Y
$$

is a submersion. The commutation relation that we must verify is precisely the condition that $T_{X, Y}$ be compatible with the mapping $\left(\rho_{Y}, \pi_{Y}\right):\left|T_{Y}\right| \cap X_{\ell+1} \rightarrow$ $\mathbb{R} \times Y$. Therefore from the generalized tubular neighborhood theorem, we get that if $X_{\ell+1}^{0}$ is an open subset of $X$ whose closure lies in $X_{\ell+1}$, then there exists $T_{X, Y}$ which satisfies the commutation relations and whose restriction to $\left|T_{Y}\right| \cap$ $X_{\ell+1}^{0}$ is isomorphic to the restriction of $T_{\ell+1}$. Now we replace $T_{Z}$ for $Z<X$ by smaller tubular neighborhoods $T_{Z}^{\prime}$ such that $X_{\ell+1}^{\prime} \subseteq X_{\ell+1}^{0}$, where $X_{\ell+1}^{\prime}$ is defined analogously to $X_{\ell+1}$, but with $T_{Z}^{\prime}$ in place of $T_{Z}$. Then $T_{X, Y}$ has the required properties.

This completes the first step: we conclude that there exists a tubular neighborhood $T_{0}$ of $X_{0}$ satisfying equation $\left(*_{0}\right)$ for any $Y<X$.

Second step. From equation $\left(*_{0}\right)$ it follows that we may assume that $T_{0}$ is compatible with $f$. For, by replacing $T_{0}$ with a smaller tubular neighborhood if necessary, we may assume that if $m \in\left|T_{0}\right|$, then for some $Y<X$, we have $m \in\left|T_{Y}\right|$ and $\pi_{0}(m) \in\left|T_{Y}\right|$. Then

$$
f \pi_{0}(m)=f \pi_{Y} \pi_{0}(m)=f \pi_{Y}(m)=f(m) .
$$


Since $T_{0}$ is compatible with $f$, we may extend a suitable restriction of $T_{0}$ to a tubular neighborhood $T$ of $X$ which is compatible with $f$, by the generalized tubular neighborhood theorem. Then, by replacing the $T_{Y}$ with possibly smaller tubular neighborhoods (as in Step 1), we get that the compatibility conditions are satisfied.

This completes the construction of $T_{X}$, and therefore also completes the proof of the proposition.

\section{Abstract Stratified Sets}

If $V$ is a closed subset of a manifold $M$ which admits a Whitney stratification (in the sense defined in Section (5) then we can find control data for this stratification by the previous section. This provides $V$ with considerable structure. The purpose of this section is to axiomatise the sort of structure which occurs. We depart only slightly from Thom's notion of abstract stratified set (3] and [4]).

Definition 8.1. An abstract stratified set is a triple $(V, \mathcal{S}, \mathfrak{J})$ satisfying the following axioms, (A1)-(A9).

(A1) $V$ is a Hausdorff, locally compact topological space with a countable basis for its topology.

This axiom implies that $V$ is metrisable. For, since $V$ is locally compact, it is regular, so the metrisability of $V$ follows from Urysohn metrisation theorem (Kelly [1.) Since $V$ is metrisable, every subset $X$ of $V$ is normal (in the sense that any two disjoint closed subsets of $X$ can be separated by open sets). We will often use this fact without explicit mention.

(A2) $\mathcal{S}$ is a family of locally closed subsets of $V$, such that $V$ is the disjoint union of the members of $\mathcal{S}$.

The members of $\mathcal{S}$ will be called the strata of $V$.

(A3) Each stratum of $V$ is a topological manifold (in the induced topology), provided with a smoothness structure (of class $C^{\mu}$ ).

(A4) The family $\mathcal{S}$ is locally finite.

(A5) The family $\mathcal{S}$ satisfies the axiom of the frontier: if $X, Y \in \mathcal{S}$ and $Y \cap \bar{X} \neq \varnothing$, then $Y \subseteq \bar{X}$.

If $Y \subseteq \bar{X}$ and $Y \neq X$, we write $Y<X$. This relation is obviously transitive: $Z<Y$ and $Y<X$ imply $Z<X$.

(A6) $\mathfrak{J}$ is a triple $\left\{\left(T_{X}\right),\left(\pi_{X}\right),\left(\rho_{X}\right)\right\}$, where for each $X \in \mathcal{S}, T_{X}$ is an open neighborhood of $X$ in $V, \pi_{X}$ is a continuous retraction of $T_{X}$ onto $X$, and $\rho_{X}: X \rightarrow[0, \infty)$ is a continuous function.

We will call $T_{X}$ the tubular neighborhood of $X$ (with respect to the given structure of a stratified set on $V), \pi_{X}$ the local retraction of $T_{X}$ onto $X$ and $\rho_{X}$ the tubular function of $X$.

(A7) $X=\left\{v \in T_{X}: \rho_{X}(v)=0\right\}$.

If $X$ and $Y$ are any strata, we let $T_{X, Y}=T_{X} \cap Y, \pi_{X, Y}=\pi_{X} \mid T_{X, Y}$, and $\rho_{X, Y}=\rho_{X} \mid T_{X, Y}$. Then $\pi_{X, Y}$ is a mapping of $T_{X, Y}$ into $X$ and $\rho_{X, Y}$ is a mapping $T_{X, Y}$ into $(0, \infty)$. Of course, $T_{X, Y}$ may be empty, in which case these are the empty mappings. 
(A8) For any strata $X$ and $Y$ the mapping

$$
\left(\pi_{X, Y}, \rho_{X, Y}\right): T_{X, Y} \rightarrow X \times(0, \infty)
$$

is a smooth submersion.

This implies $\operatorname{dim} X<\operatorname{dim} Y$ when $T_{X, Y} \neq \varnothing$.

(A9) For any strata $X, Y$, and $Z$, we have

$$
\begin{aligned}
& \pi_{X, Y} \pi_{Y, Z}(v)=\pi_{X, Z}(v), \\
& \rho_{X, Y} \pi_{Y, Z}(v)=\rho_{X, Z}(v)
\end{aligned}
$$

whenever both sides of this equation are defined, i.e., whenever $v \in T_{X, Z} \cap$ $T_{Y, Z}$ and $\pi_{Y, Z}(v) \in T_{X, Y}$.

Definition 8.2. We say that two stratified sets $(V, \mathcal{S}, \mathfrak{J})$ and $\left(V^{\prime}, \mathcal{S}^{\prime}, \mathfrak{J}^{\prime}\right)$ are equivalent if the following conditions hold.

(a) $V=V^{\prime}, \mathcal{S}=\mathcal{S}^{\prime}$, and for each stratum $X$ of $\mathcal{S}=\mathcal{S}^{\prime}$, the two smoothness structures on $X$ given by the two stratifications are the same.

(b) If $\mathfrak{J}=\left\{\left(T_{X}\right),\left(\pi_{X}\right),\left(\rho_{X}\right)\right\}$ and $\mathfrak{J}^{\prime}=\left\{\left(T_{X}^{\prime}\right),\left(\pi_{X}^{\prime}\right),\left(\rho_{X}^{\prime}\right)\right\}$, then for each stratum $X$, there exists a neighborhood $T_{X}^{\prime \prime}$ of $X$ in $T_{X} \cap T_{X}^{\prime}$ such that $\rho_{X}\left|T_{X}^{\prime \prime}=\rho_{X}^{\prime}\right| T_{X}^{\prime \prime}$ and $\pi_{X}\left|T_{X}^{\prime \prime}=\pi_{X}^{\prime}\right| T_{X}^{\prime \prime}$.

From the normality of arbitrary subsets of a stratified set, it follows that any (abstract) stratified set is equivalent to one which satisfies the following conditions.

(A10) If $X, Y$ are strata and $T_{X, Y} \neq \varnothing$, then $X<Y$.

(A11) If $X, Y$ are strata and $T_{X} \cap T_{Y} \neq \varnothing$, then $X$ and $Y$ are comparable, i.e., one of the following holds: $X<Y, Y<X$, or $X=Y$.

From $(A 10)$ it follows that $X<Y$ if and only if $T_{X, Y} \neq \varnothing$, and from (A11) that $X$ and $Y$ are comparable if and only if $T_{X} \cap T_{Y} \neq \varnothing$.

Note that from $(A 8)$ it follows that the relation $X<Y$ defines a partial order on $\mathcal{S}$. It is enough to verify $X<Y$ and $Y<X$ do not hold simultaneously. But (A8) implies $X<Y \Rightarrow \operatorname{dim} X<\operatorname{dim} Y$.

As an example of an (abstract) stratified set, let $V$ be a subset of a manifold $M$ and suppose $V$ admits a Whitney stratification $\mathcal{S}$, and let $\left\{T_{X}^{\prime}\right\}$ be a family of control data for $\mathcal{S}$. Let $T_{X}=T_{X}^{\prime} \cap V, \pi_{X}=\pi_{X}^{\prime} \mid T_{X}$, and $\rho_{X}=\rho_{X}^{\prime} \mid T_{X}$. Set $\mathfrak{J}=\left\{T_{X}\right\}$. Then $(V, \mathcal{S}, \mathfrak{J})$ is an abstract stratified set. In this way, we associate with any system of control data for a Whitney stratified set $V$ a structure of an abstract stratified set on $V$.

Hence it follows from Proposition 7.1 that any Whitney stratified set admits the structure of an abstract stratified set.

If $(V, \mathcal{S}, \mathfrak{J})$ is a stratified set, $V^{\prime}$ is any topological space, and $\varphi: V^{\prime} \rightarrow V$ is a homeomorphism, then the structure of a stratified set on $V$ "pulls back" in an obvious way to give a structure of a stratified set $\left(V^{\prime}, \varphi^{*} \mathcal{S}, \varphi^{*} \mathfrak{J}\right)$ on $V^{\prime}$.

If $\left(V^{\prime}, \mathcal{S}^{\prime}, \mathfrak{J}^{\prime}\right)$ and $(V, \mathcal{S}, \mathfrak{J})$ are abstract stratified sets, then a homeomorphism $\varphi: V^{\prime} \rightarrow V$ is said to be an isomorphism of stratified sets if $\left(V^{\prime}, \mathcal{S}^{\prime}, \mathfrak{J}^{\prime}\right)$ is equivalent to $\left(V^{\prime}, \varphi^{*} \mathcal{S}, \varphi^{*} \mathfrak{J}\right)$

The uniqueness result that we will prove below implies the following: if $\mathcal{S}$ is a Whitney stratification of a subset $V$ of a manifold, and $\mathfrak{J}$ and $\mathfrak{J}^{\prime}$ are two system of control data, then the abstract stratified sets $(V, \mathcal{S}, \mathfrak{J})$ and $\left(V, \mathcal{S}, \mathfrak{J}^{\prime}\right)$ are isomorphic. 


\section{Controlled vector fields}

Throughout this section, we let $(V, \mathcal{S}, \mathfrak{J})$ be an (abstract) stratified set. We suppose $\mu \geq 2$.

Definition. By a stratified vector field $\eta$ on $V$, we mean a collection $\left\{\eta_{X}: X \in \mathcal{S}\right\}$, where for each stratum $X$, we have that $\eta_{X}$ is a smooth vector field on $X$.

By smooth vector field we mean a vector field of class $C^{\mu-1}$.

Let $\mathfrak{J}=\left\{\left(T_{X}\right),\left(\pi_{X}\right),\left(\rho_{X}\right)\right\}$, and for two strata $X$ and $Y$, let $T_{X, Y}, \pi_{X, Y}$, and $\rho_{X, Y}$ be defined as in the previous section.

Definition. A stratified vector field $\eta$ on $V$ will be said to be controlled (by $\mathfrak{J}$ ) if the following control conditions are satisfied: for any stratum $Y$ there exists a neighborhood $T_{Y}^{\prime}$ of $Y$ in $T_{Y}$ such that for any second stratum $X>Y$ and any $v \in T_{Y}^{\prime} \cap X$, we have

$$
\begin{aligned}
\eta_{X} \rho_{Y, X}(v) & =0, \\
\left(\pi_{Y, X}\right)_{*} \eta_{X}(v) & =\eta_{Y}\left(\pi_{Y, X}(v)\right) .
\end{aligned}
$$

Definition. If $P$ is a smooth manifold and $f: V \rightarrow P$ is a continuous mapping, we will say that $f$ is a controlled submersion if the following conditions are satisfied.

(1) $f \mid X: X \rightarrow P$ is a smooth submersion, for each stratum $X$ of $V$.

(2) For any stratum $X$, there is a neighborhood $T_{X}^{\prime}$ of $X$ in $T_{X}$ such that $f(v)=$ $f \pi_{X}(v)$ for all $v \in T_{X}^{\prime}$.

Note that both the notions that we have just introduced depend only on the equivalence class of the stratified set $(V, \mathcal{S}, \mathfrak{J})$, i.e., if $\left(V, \mathcal{S}, \mathfrak{J}^{\prime}\right)$ is a stratified set which is equivalent to $(V, \mathcal{S}, \mathfrak{J})$, then a controlled vector field (or controlled submersion) with respect to one of these stratified sets is the same as a controlled vector field (or controlled submersion) with respect to the other.

Proposition 9.1. If $f: V \rightarrow P$ is a controlled submersion, then for any smooth vector field $\zeta$ on $P$, there is a controlled vector field $\eta$ on $V$ such that $f_{*} \eta(v)=$ $\zeta(f(v))$ for all $v \in V$.

Proof. By induction on the dimension of $V$ (where the dimension of $V$ is defined to be the supremum of the dimensions of the strata of $V$ ). By the $k$ skeleton $V_{k}$ of $V$, we will mean the union of all strata of $V$ of dimension $\leq k$. Clearly $V_{k}$ has the structure of a stratified set, where the strata of $V_{k}$ are the strata of $V$ which lie in $V_{k}$, the tubular neighborhoods are the intersections with $V_{k}$ of the tubular neighborhoods (in $V$ ) of strata in $V_{k}$ and the local retractions and tubular functions on $V_{k}$ are the restrictions of the local retractions and tubular functions on $V$.

In the case $\operatorname{dim} V=0$, the statement of the proposition is trivial. Hence, by induction, it is enough to show that if the proposition is true whenever $\operatorname{dim} V \leq k$ then it is true when $\operatorname{dim} V=k+1$. Thus, we may (and do) assume that $\operatorname{dim} V=$ $k+1$ and that there is a controlled vector field $\eta_{k}$ on $V_{k}$ such that $f_{*} \eta_{k}(v)=\zeta(f(v))$ for all $v \in V_{k}$. We will show that there exists a controlled vector field $\eta$ on $V$ which extends $\eta_{k}$ such that $f_{*} \eta(v)=\zeta(f(v))$ for all $v \in V$.

To construct $\eta$, it is enough to construct $\eta_{X}$ separately for each stratum $X$ of $V$ such that $\operatorname{dim} X=k+1$, because the condition that a vector field be controlled involves only strata $Y, X$ such that $Y<X$. 
Since by the induction assumption $\eta_{k}$ is controlled, we can choose neighborhoods $T_{Y}^{1}$ of $Y$ in $T_{Y}$ (one for each stratum $Y \subseteq V_{k}$ ) such that if $Y<Z$ are strata, then the control conditions (9.1) and (9.2) are satisfied (with $Z$ in place of $X$ ) for $v \in T_{Y}^{1} \cap Z$. By the assumption that $f$ is controlled, we may choose the neighborhoods $T_{Y}^{1}$ such that $f(v)=f \pi_{Y}(v)$ for all $v \in T_{Y}^{1}$.

Is is easily seen that we may choose neighborhoods $T_{Y}^{2}$ of $Y$ in $T_{Y}^{1}$ (one for each stratum $Y \subseteq V_{k}$ ) such that the following holds: if $Y<Z$ are strata in $V_{k}$ then

$$
\pi_{Z}\left(T_{Y}^{2} \cap T_{Z}^{2}\right) \subseteq T_{Y}^{1} .
$$

We can furthermore choose the $T_{Y}^{2}$ so that $T_{Y}^{2}$ is closed in $V-\partial Y$ (where $\partial Y$ denotes the frontier of $Y$ ), since $V-\partial Y$ is metrisable and therefore normal, and $Y$ is closed in $V-\partial Y$. Finally, we can choose the $T_{Y}^{2}$ so that if $Y$ is not comparable to $Z$, then $T_{Y}^{2} \cap T_{Z}^{2}=\varnothing$.

Now consider the following conditions on a vector field $\eta_{X}$ on $X$ :

(9.3Y) The control conditions (9.1) and (9.2) are satisfied for any $v \in T_{Y}^{2} \cap X$.

(9.4) $\quad f_{*} \eta_{X}(v)=\zeta(f(v))$ for all $v \in X$.

We claim that there is a vector field $\eta_{X}$ on $X$ satisfying (9.4) and (9.3 ) for all strata $Y<X$. To prove this claim will clearly be enough to prove the proposition.

Consider a point $v \in X$. The set $\mathcal{S}_{v}$ of strata $Y<X$ such that $v \in T_{Y}^{2}$ is totally ordered, since if $Y$ and $Z$ are not comparable then $T_{Y}^{2} \cap T_{Z}^{2}=\varnothing$. If $\mathcal{S}_{v}$ is not empty, then there is a largest member $Y=Y_{v}$.

Suppose for the moment this is the case and $\left(9.3_{Y}\right)$ holds at $v$. Then $\left(9.3_{Z}\right)$ holds for all $Z \in \mathcal{S}_{v}$. For, either $Z=Y$ or $Z<Y$. In the latter case $\pi_{Y}(v) \in T_{Z}^{1}$ (by the choice of the $T_{Y}^{2}$ 's). Then

$$
\begin{aligned}
\eta_{X} \rho_{Z, X}(v) & =\eta_{X} \rho_{Z, Y} \pi_{Y, X}(v) \\
& =\left(\pi_{Y, X}\right)_{*} \eta_{X}(v) \rho_{Z, Y} \\
& =\eta_{Y}\left(\pi_{Y, X}(v)\right) \rho_{Z, Y}=0
\end{aligned}
$$

and

$$
\begin{aligned}
\left(\pi_{Z, X}\right)_{*} \eta_{X}(v) & =\left(\pi_{Z, Y}\right)_{*}\left(\pi_{Y, X}\right)_{*} \eta_{X}(v) \\
& =\left(\pi_{Z, Y}\right)_{*} \eta_{Y}\left(\pi_{Y, X}(v)\right) \\
& =\eta_{Z}\left(\pi_{Z, Y} \pi_{Y, X}(v)\right) \\
& =\eta_{Z}\left(\pi_{Z, X}(v)\right) .
\end{aligned}
$$

Thus $\left(9.3_{Z}\right)$ holds at $v$ for all $Z \in \mathcal{S}_{v}$. Furthermore

$$
\begin{aligned}
f_{*} \eta_{X}(v) & =\left(f \circ \pi_{Y, X}\right)_{*} \eta_{X}(v) \\
& =f_{*} \eta_{Y}\left(\pi_{Y, X}(v)\right) \\
& =\zeta(f(v)) .
\end{aligned}
$$

Thus (9.4) holds at $v$.

This shows that to construct $\eta_{X}$ satisfying (9.4) and $9.3_{Y}$ for all $Y<X$, it is enough to construct $\eta_{X}$ satisfying $\left(9.3_{Y_{v}}\right)$ at $v$ for all $v \in X$ for which $\mathcal{S}_{v}$ is nonempty, and satisfying (9.4) at $v$ for all $v \in X$ for which $\mathcal{S}_{v}$ is empty. Clearly, we can construct a vector field $\eta_{X}$ in a neighborhood of each point $v$ in $X$ satisfying the appropriated condition $\left(9.3_{Y_{v}}\right)$ or (9.4). Since the set of vectors satisfying the 
appropriate condition in $T X_{v}$ is convex, we may construct $\eta_{X}$ globally by means of a partition of unity.

\section{ONE PARAMETER GROUPS}

Let $V$ be a topological space. By a one-parameter group of homeomorphism of $V$, we mean a continuous mapping $\alpha: \mathbb{R} \times V \rightarrow V$ such that $\alpha_{t+s}(v)=\alpha_{t} \alpha_{s}(v)$ for all $t, s \in \mathbb{R}$ and all $v \in V$, and $\alpha_{0}(v)=v$ for all $v \in V$. Now suppose $V$ is a stratified set $(V, \mathcal{S}, \mathfrak{J})$ and $\alpha$ preserves each stratum. If $\eta$ is a stratified vector field on $V$, we say that $\eta$ generates $\alpha$ if the following condition is satisfied. For any $v \in V$, the mapping $t \mapsto \alpha_{t}(v)$ of $\mathbb{R}$ into $V$ is $C^{1}$ (as a mapping into the stratum which contains $v$ ) and

Note that this implies

$$
\left.\frac{d}{d t}\left(\alpha_{t}(v)\right)\right|_{t=0}=\eta(v) .
$$

$$
\frac{d}{d t}\left(\alpha_{t}(v)\right)=\pi\left(\alpha_{t}(v)\right), \quad t \in \mathbb{R} .
$$

It is well known that any $C^{1}$ vector field on a compact manifold without boundary generates a unique one-parameter group (see, e.g., [2, p. 66]). It is also known that to extend this result to noncompact manifolds, we must generalize the notion of one-parameter group.

Definition. Let $V$ be a locally compact space. A local one-parameter group (on $V$ ) is a pair $(J, \alpha)$, where $J$ is an open subset of $\mathbb{R} \times V$ and $\alpha: J \rightarrow V$ is a continuous mapping such that the following hold.

(a) $0 \times V \subseteq J$ and $\alpha(0, v)=v$ for all $v \in V$.

(b) If $v \in V$, then the set $J_{v}=J \cap(\mathbb{R} \times v) \subseteq \mathbb{R}$ is an open interval $\left(a_{v}, b_{v}\right)$, possibly infinite at one or both ends.

(c) If $v \in V$, and $t, s$, and $t+s$ are in $\left(a_{v}, b_{v}\right)$ then $\alpha(t+s, v)=\alpha(t, \alpha(s, v))$.

(d) For any $v \in V$ and any compact set $K \subseteq V$, there exists $\varepsilon>0$ such that $\alpha(t, v) \notin K$ if $t \in\left(a_{v}, a_{v}+\varepsilon\right) \cup\left(b_{v}-\varepsilon, b_{v}\right)$.

From now on in this section, we suppose $(V, \mathcal{S}, \mathfrak{J})$ is an (abstract) stratified set, and $\eta$ is a stratified vector field on $V$.

Definition. If $(J, \alpha)$ is a local one-parameter group (on $V$ ), we say $\eta$ generates $\alpha$ if the following conditions (国) -(드) are satisfied.

(a) Each stratum $X$ of $V$ is invariant under $\alpha$, i.e., $\alpha[J \cap(\mathbb{R} \times X)] \subseteq X$.

(b) For each $v \in V$, the mapping $t \mapsto \alpha(t, v)$ of $\left(a_{v}, b_{v}\right)$ into the stratum which contains $v$ is $C^{1}$.

(c) For any $v \in V$, we have

$$
\left.\frac{d}{d t} \alpha(t, v)\right|_{t=0}=\eta(v) .
$$

Since $\alpha$ is a local one-parameter group, condition (ㄷ) is equivalent to:

$\left(\mathrm{c}^{\prime}\right)$ For any $(t, v) \in V$, we have

$$
\frac{d}{d t} \alpha(t, v)=\eta(\alpha(t, v)) .
$$


This generalizes the ordinary notion of what it means for a vector field to generate a local one parameter group.

Since $(V, \mathcal{S}, \mathfrak{J})$ is a stratified set, it makes sense to talk of a controlled vector field on $V$ (Section 9).

Proposition 10.1. If $\eta$ is a controlled vector field on $V$, then $\eta$ generates a unique local one parameter group $(J, \alpha)$.

Proof. For each stratum $X$, the restriction $\eta_{X}$ of $\eta$ to $X$ is a smooth vector field on $X$ (by the definition of stratified vector field); hence $\eta_{X}$ generates a unique smooth local one-parameter group $\left(J_{X}, \alpha_{X}\right)$ of diffeomorphisms of $X$, by a standard result in differential geometry [2, IV, Section 2]. Let $(J, \alpha)$ be defined by

$$
\begin{aligned}
& J=\bigcup_{X \in \mathcal{S}} J_{X}, \\
& \alpha=\bigcup_{X \in \mathcal{S}} \alpha_{X} .
\end{aligned}
$$

We assert that $(J, \alpha)$ is a local one-parameter group generated by $\eta$.

It is clear that (国), (b), and (ㄷ) in the definition of local one-parameter group hold, and that if $\alpha$ is a local one-parameter group, then it is generated by $v$. Uniqueness is obvious since each $\left(J_{X}, \alpha_{X}\right)$ is unique. All that remains to be verified is that $J$ is open, $\alpha$ is continuous, and (d) holds.

We begin by showing that (d) holds. If not, there exists $v \in V$ and a compact set $K$ in $V$ such that $\alpha(t, v) \in K$ for values of $t$ arbitrarily close to $a_{v}$ or $b_{v}$. We may suppose that $\alpha(t, v) \in K$ for values of $t$ arbitrarily close to $b_{v}$; the other case is treated similarly. Then there exists a sequence $\left\{t_{i}\right\}$, converging to $b_{v}$ from below, such that $y=\lim \alpha\left(t_{i}, v\right)$ exists and lies in $K$. Let $X$ (resp. $Y$ ) denote the stratum of $V$ which contains $v$ (resp. $y$ ).

If $X=Y$, we get a contradiction to the fact that $\alpha_{X}$ is a one-parameter group. Otherwise $Y<X$. For large $i, \rho_{Y, X}\left(\alpha_{v}\left(t_{i}\right)\right)$ and $\pi_{Y, X}\left(\alpha_{v}\left(t_{i}\right)\right)$ are defined, and the control conditions are satisfied for $m_{i}=\alpha_{v}\left(t_{i}\right)$.

Thus, by taking $i$ sufficiently large, we may suppose that there exists $\varepsilon>t-t_{i}$ such that $[0, \varepsilon] \subseteq J_{y_{i}}$, where $y_{i}=\pi_{Y, X}\left(m_{i}\right)$, and if $T_{Y}$ is the tubular neighborhood of $Y, \pi_{Y}$ is the local retraction of $T_{Y}$ onto $Y$, and $\rho_{Y}$ is the tubular function of $Y$, then $\rho_{Y, X}\left(m_{i}\right)<\varepsilon_{Y}$ on $\alpha_{y_{i}}([0, \varepsilon])$ and the control conditions for the pair $Y, X$ are satisfied for

$$
m \in\left\{\rho_{Y, X}=\rho_{Y, X}\left(m_{i}\right)\right\} \cap \pi_{Y, X}^{-1}\left(\alpha_{y_{i}}[0, \varepsilon]\right) \cap X .
$$

Since $\left\{\rho_{Y, X}=\rho_{Y, X}\left(m_{i}\right)\right\} \cap \pi_{Y, X}^{-1}\left(\alpha_{y_{i}}[0, \varepsilon]\right)$ is compact (because $\rho_{Y, X}\left(m_{i}\right)<\varepsilon_{Y}$ on $\left.\alpha_{y_{i}}([0, \varepsilon])\right)$, and $\alpha_{v}$ stays in $X$ (by definition), it follows from the control conditions that

$$
\alpha_{v}\left(t_{i}+s\right) \in\left\{\rho_{Y, X}=\rho_{Y, X}\left(m_{i}\right)\right\} \cap \pi_{Y, X}^{-1}\left(\alpha_{y_{i}}(s)\right) \cap X \quad \text { for } \quad 0 \leq s \leq \varepsilon .
$$

But this contradicts the hypothesis that $\alpha_{v}\left(t_{j}\right) \rightarrow y$ as $j \rightarrow \infty$. This contradiction proves (d).

Now let $(t, v) \in J$. We will show that $J$ is a neighborhood of $(t, v)$ and $\alpha$ is continuous at $(t, v)$. We will suppose $t \geq 0$; the other case is treated similarly. As before, let $X$ be the stratum which contains $v$. Since $\alpha_{X}$ is a local one-parameter group, there is a compact neighborhood $U$ of $v$ in $X$ and an $\varepsilon>0$ such that $[-\varepsilon, t+\varepsilon] \times U \subseteq J$. Let $T_{X}$ denote the tubular neighborhood of $X, \pi_{X}$ the local 
retraction of $T_{X}$ on $X$, and $\rho_{X}$ the tubular function of $X$. Since $\alpha_{X}([-\varepsilon, t+\varepsilon] \times U)$ is compact, we may choose an $\varepsilon_{1}>0$ such that the following hold:

(a) Let $\Sigma=\left\{y \in T_{X}: \rho_{X}(y) \leq \varepsilon_{1}\right.$ and $\left.\pi_{X}(y) \in \alpha_{X}([-\varepsilon, t+\varepsilon] \times U)\right\}$. Then $\Sigma$ is compact.

(b) If $y \in \Sigma$, then the control conditions for the pair $X, Y$ hold at $y$, where $Y$ is the stratum which contains $y$.

Clearly, the set $\Sigma_{0}$ of $y \in T_{X}$ such that $\rho_{X}(y) \leq \varepsilon_{1}$ and $\pi_{X}(y) \in U$ is a neighborhood of $v$ in $V$. If $y \in \Sigma_{0}$, it follows from the control conditions that

$$
\begin{aligned}
& \rho_{X}\left(\alpha_{y}(s)\right)=\rho_{X}(y), \\
& \pi_{X}\left(\alpha_{y}(s)\right)=\alpha_{\pi_{X}(y)}(s)
\end{aligned}
$$

for all $s \in J_{y}$ such that $\alpha_{y}\left(s^{\prime}\right) \in \Sigma$ for $0 \leq s^{\prime}<s$. From these facts and (d), it follows that $[-\varepsilon, t+\varepsilon] \times \Sigma_{0} \subseteq J$; thus $J$ contains a neighborhood of $(t, v)$.

The argument that we have just given shows that if $\left(t^{\prime}, y\right) \in[t-\varepsilon, t+\varepsilon] \times \Sigma_{0}$, then $y^{\prime}=\alpha\left(t^{\prime}, y\right) \in T_{X}, \rho_{X}\left(y^{\prime}\right) \leq \varepsilon_{1}$, and $\pi_{X}\left(y^{\prime}\right)=\alpha\left(t^{\prime}, \pi_{X}(y)\right)$. Hence, for an arbitrarily small neighborhood of $\alpha(t, x)$ we may choose $\varepsilon>0$ and a neighborhood $\Sigma_{1}$. Hence, $\alpha$ is continuous at $(t, v)$.

Corollary 10.2. Let $P$ be a manifold, and $f: V \rightarrow P$ be a proper, controlled submersion. Then $f$ is a locally trivial fibration.

Proof. It is enough to consider the case when $P=\mathbb{R}^{k}$ and show in this case that there is a homeomorphism $h: V \rightarrow V_{0} \times \mathbb{R}^{k}$, where $V_{0}$ denotes the fibre of $V$ over 0 , such that the following diagram commutes:

(Diagram 10.1)

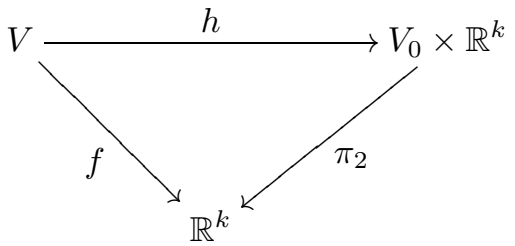

where $\pi_{2}$ denotes the projection on the second factor.

Consider the coordinate vector fields $\partial_{1}, \cdots, \partial_{k}$ on $\mathbb{R}^{k}$. By Proposition 9.1, for each $i, 1 \leq i \leq k$, there is a controlled vector field $\tilde{\partial}_{i}$ on $V$ such that

$$
f_{*} \tilde{\partial}_{i}(v)=\partial_{i}(f(v)), \quad v \in V .
$$

By Proposition 10.1, each $\partial_{i}$ generates a local one-parameter group $\left(J_{i}, \alpha_{i}\right)$. Clearly

$$
f\left(\alpha_{i}(t, v)\right)=f(v)+(0, \cdots, 0, t, 0, \cdots, 0),
$$

where the non-vanishing entry is in the $i$-th place. Then from the assumption that $f$ is proper and condition (d) in the definition of a one parameter group, it follows that $J_{i}=\mathbb{R} \times V$. Let $h$ be given by

$$
h(v)=\left(\alpha_{1}\left(-t_{1}, \alpha_{2}\left(-t_{2}, \cdots, \alpha_{k}\left(-t_{k}, v\right) \cdots\right)\right), f(v)\right)
$$

where we set $f(v)=\left(t_{1}, \cdots, t_{k}\right)$. It is easily seen that $h$ maps $V$ into $V_{0} \times \mathbb{R}^{k}$ and that Diagram 10.1 commutes. Let $\bar{h}: V_{0} \times \mathbb{R}^{k} \rightarrow V$ be defined by

$$
\bar{h}\left(v,\left(t_{1}, \cdots, t_{k}\right)\right)=\alpha_{k}\left(t_{k}, \cdots, \alpha_{2}\left(t_{2}, \alpha_{1}\left(t_{1}, v\right) \cdots\right)\right) .
$$

From the fact that the $\alpha_{i}$ 's are one-parameter groups, it follows that $h \bar{h}=\bar{h} h=$ identity. Hence $h$ is a homeomorphism, as required. 
Note that $V_{0}$ has a natural structure of a stratified set $\left(V_{0}, \mathcal{S}_{0}, \mathfrak{J}_{0}\right)$, where $\mathcal{S}_{0}$ and $\mathfrak{J}_{0}$ are defined as follows. $\mathcal{S}_{0}$ is the collection $\left\{X \cap V_{0}: X \in \mathcal{S}\right\}$. If $X \in \mathcal{S}$ and $X_{0}=X \cap V_{0}$ is the corresponding member of $\mathcal{S}_{0}$, then we let $T_{X_{0}}=T_{X} \cap V_{0}$, $\pi_{X_{0}}=\pi_{X} \mid T_{X_{0}}$ and $\rho_{X_{0}}=\rho_{X} \mid T_{X_{0}}$. Note that $\pi_{X_{0}}$ maps into $X_{0}$ because $f$ is a controlled submersion. We let $\mathfrak{J}_{0}$ be the triple $\left\{\left(T_{X_{0}}\right),\left(\pi_{X_{0}}\right),\left(\rho_{X_{0}}\right)\right\}$.

Furthermore $V_{0} \times \mathbb{R}^{k}$ has a structure of a stratified set (defined in an obvious way).

Corollary 10.3. If $h$ is constructed as in the proof of Corollary 10.2, then $h$ is an isomorphism of stratified sets.

Proof. Immediate from the construction of $h$. (See the end of Section 8 for the definition of isomorphism.)

Corollary 10.4. Let $M$ be a manifold, let $S$ be a closed subset of $M$ and let $\mathcal{S}$ be a Whitney stratification of $S$. Let $X$ and $Y$ be strata with $X<Y$. Let $W$ be a submanifold of $M$ which meets $X$ transversally. Then $X \cap W \subseteq \overline{Y \cap W}$.

Proof. Let $x \in X \cap W$. To show $x \in \overline{Y \cap W}$, it is enough to consider what happens in a neighborhood of $x$. By replacing $M$ with a sufficiently small neighborhood of $x$, we may suppose that $X$ is connected and closed, and there exists a tubular neighborhood $T_{X}$ of $X$ in $M$ such that $W \cap\left|T_{X}\right|=\pi_{X}^{-1}(W \cap X)$, where $\pi_{X}:\left|T_{X}\right| \rightarrow$ $X$ is the projection associated to $T_{X}$. From Lemma 7.3 it follows that by choosing $T_{X}$ sufficiently small, we may suppose that there exists $\varepsilon>0$ such that $\rho_{X}<\varepsilon$ on $T_{X}$, where $\rho_{X}$ is the tubular function associated to $T_{X}$, where $\left(\rho_{X}, \pi_{X}\right):\left|T_{X}\right| \rightarrow$ $[0, \varepsilon) \times X$ is proper, and where for each stratum $Z$ of $\mathcal{S}$, the mapping

$$
\left(\rho_{X}, \pi_{X}\right)|Z: Z \cap| T_{X} \mid \rightarrow(0, \varepsilon) \times X
$$

is a submersion.

Let $\mathcal{S}^{\prime}=\left\{Z \cap\left(\left|T_{X}\right|-X\right): Z \in \mathcal{S}\right\}$. Then $\mathcal{S}^{\prime}$ is a Whitney stratification of $S \cap\left(\left|T_{X}\right|-X\right)$. By Proposition 10.1, there is a family of control data $\mathfrak{J}^{\prime}$ for $\mathcal{S}^{\prime}$ which is compatible with $\left(\rho_{X}, \pi_{X}\right)$. Then $\left(S \cap\left(\left|T_{X}\right|-X\right), \mathcal{S}^{\prime}, \mathfrak{J}^{\prime}\right)$ is an abstract stratified set and $\left(\rho_{X}, \pi_{X}\right)$ is a controlled submersion. Hence by Corollary 10.2. $S \cap\left(\left|T_{X}\right|-X\right)$ is a locally trivial bundle over $(0, \varepsilon) \times X$, and by Corollary 10.3, the local trivializations respect the stratification.

It follows that any stratum of $\mathcal{S}^{\prime}$ (e.g., $\left.Y \cap\left(\left|T_{X}\right|-X\right)\right)$ intersects each fibre of $\left(\rho_{X}, \pi_{X}\right)$. In particular $\varnothing \neq Y \cap\left(\rho_{X}, \pi_{X}\right)^{-1}\left(\varepsilon^{\prime}, X\right) \subseteq Y \cap W$ for $0<\varepsilon^{\prime}<\varepsilon$. It follows that $x \in \overline{Y \cap W}$.

The next corollary says that a stratification which satisfies all the conditions of a Whitney stratification except the condition of the frontier also satisfies the condition of the frontier, provided that its strata are connected.

Corollary 10.5. Let $M$ be a manifold and $\mathcal{S}$ be a locally finite stratification of a closed subset $V$ of $M$ whose strata are connected such that any pair of strata satisfy condition $b$. Then $\mathcal{S}$ is a Whitney stratification.

Proof. It suffices to show that the condition of the frontier holds. Suppose $X$ and $Y$ are strata and $Y \cap \bar{X} \neq \varnothing$. The proof of Corollary 10.4 shows that $Y \cap \bar{X}$ is open in $Y$. Since $Y \cap \bar{X}$ is clearly closed in $Y$, and $Y$ is connected, this proves $Y \subseteq \bar{X}$.

The proof of Corollary 10.4 also shows: 
Corollary 10.6. Let $M$ be a manifold, $\mathcal{S}$ a Whitney stratification of $M, X a$ stratum of $M$, and $T_{X}$ a tubular neighborhood of $X$ in $M$ such that for any stratum $Z$ of $\mathcal{S}$, the mapping $\left(\rho_{X}, \pi_{X}\right):\left(\left|T_{X}\right|-X\right) \cap Z \rightarrow X_{\varepsilon}$ is a submersion, where $T_{X}=(E, \varphi, \varepsilon)$ and $X_{\varepsilon}=\{(t, x) \in \mathbb{R} \times X: 0<t<\varepsilon(x)\}$. Then the bundle $\left(\left|T_{X}\right|-X,\left(\rho_{X}, \pi_{X}\right), X_{\varepsilon}\right)$ is locally trivial and the local trivializations can be chosen to respect the stratification.

\section{The Isotopy lemmas of Thom}

In this section, we will state Thom's first and second isotopy lemmas. We will prove the first and sketch a proof of the second.

Throughout this section, we let $M$ and $P$ be smooth manifolds, $f: M \rightarrow P$ a smooth mapping, and $S$ a closed subset of $M$ which admits a Whitney stratification.

Proposition 11.1 (Thom's first isotopy lemma). Suppose $f \mid S: S \rightarrow P$ is proper and $f \mid X: X \rightarrow P$ is a submersion for each stratum $X$ of $S$. Then the bundle $(S, f, P)$ is locally trivial.

Proof. By Proposition 7.1, we can find a system of control data for $S$ which is compatible with $f$. This provides $S$ with a structure of an abstract stratified set in such a way that $f$ is a controlled submersion. Then the conclusion of the theorem is an immediate consequence of Corollary 10.2 .

Remark. Thom considered the case $P=\mathbb{R}$. If $a, b \in \mathbb{R}$, then the proof of Proposition 10.1 constructs an isotopy from the fibre $S_{a}$ to the fibre $S_{b}$, whence the name "isotopy lemma".

The second isotopy lemma is an analogous result for mappings instead of spaces. Consider a diagram of spaces and mappings:

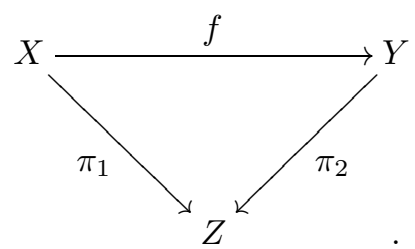

We say that $f$ is trivial over $Z$ if there exists spaces $X_{0}$ and $Y_{0}$, a mapping $f_{0}: X_{0} \rightarrow$ $Y_{0}$ and homeomorphisms $X \approx X_{0} \times Z, Y \approx Y_{0} \times Z$ such that the following diagram of spaces and mappings is commutative:

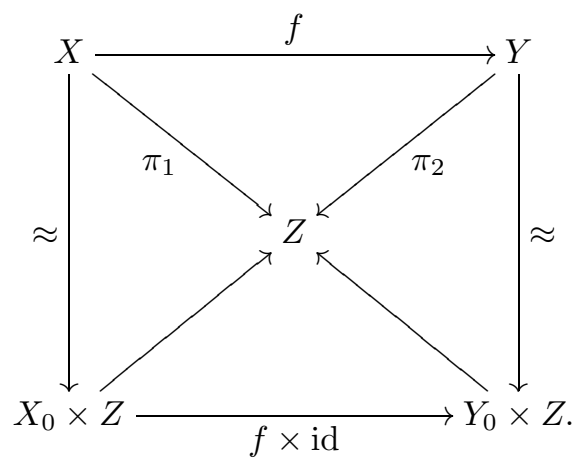


We say $f$ is locally trivial over $Z$ if for any $z \in Z$, there is a neighborhood $U$ of $z$ in $Z$ such that in the diagram

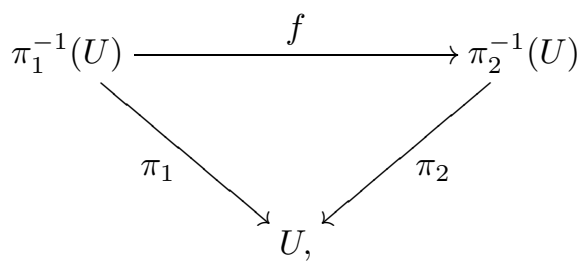

we have that $f$ is trivial over $U$.

Local triviality of a mapping $f$ over a space $Z$ has a consequence which is very important in our proof that topologically stable mappings are dense. We think of $f$ as a family $\left\{f_{a}: a \in Z\right\}$ of mappings, where $f_{a}: X_{a} \rightarrow Y_{a}$ is the mapping obtained by restricting $f$ to the fibre $X_{a}$ of $X$ over $a$. If $Z$ is connected and $f$ is locally trivial over $Z$, then for any $a$ and $b$ in $Z$, the mappings $f_{a}$ and $f_{b}$ are equivalent in the sense that there exist homeomorphisms $h: X_{a} \rightarrow X_{b}$ and $h^{\prime}: Y_{a} \rightarrow Y_{b}$ such that $h^{\prime} f_{a}=f_{b} h$.

This is the relation of equivalence that is used in the definition of topologically stable mapping, and a step in the proof that the topologically stable mappings form an open dense set will be to show that certain families of mappings are locally trivial in the sense defined above, by an application of Thom's second isotopy lemma.

Now suppose $M^{\prime}$ is a smooth manifold and $S^{\prime}$ is a closed subset of $M^{\prime}$, which admits a Whitney stratification $\mathcal{S}^{\prime}$. Let $g: M^{\prime} \rightarrow M$ be a smooth mapping and suppose $g\left(S^{\prime}\right) \subseteq S$. Thom's second isotopy lemma gives sufficient conditions for the following diagram to be locally trivial:

(Diagram 11.1)

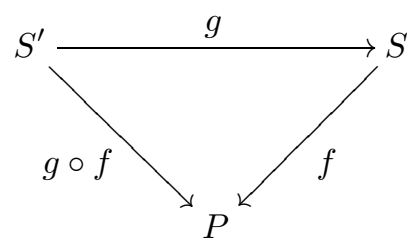

To state Thom's second isotopy lemma, we must introduce Thom's condition $a_{g}$. Let $X$ and $Y$ be submanifolds of $M^{\prime}$ and let $y$ be a point in $Y$. Suppose $g \mid X$ and $g \mid Y$ are of constant rank. We say the pair $(X, Y)$ satisfies condition $a_{g}$ at $y$ if the following holds:

Let $\left\{x_{i}\right\}$ be any sequence of points in $X$ converging to $y$. Suppose that sequence of planes $\operatorname{ker}\left(d\left(g \mid X^{\prime}\right)_{x_{i}}\right) \subseteq T M_{x_{i}}^{\prime}$ converges to a plane $\tau \subseteq T M_{y}^{\prime}$ in the appropriate Grassmannian bundle. Then $\operatorname{ker}\left(d\left(g \mid Y^{\prime}\right)_{y}\right) \subseteq \tau$.

We say the pair $(X, Y)$ satisfies condition $a_{g}$ if it satisfies condition $a_{g}$ at every point $y$ of $Y$.

Now, we return to the situation of Diagram 11.1. We will say that $g$ is a Thom mapping (over $P$ ) if the following conditions are satisfied.

(a) $g \mid S^{\prime}$ and $f \mid S$ are proper.

(b) For each stratum $X$ of $\mathcal{S}, f \mid X$ is a submersion.

(c) For each stratum $X^{\prime}$ of $\mathcal{S}^{\prime}, g\left(X^{\prime}\right)$ lies in a stratum $X$ of $\mathcal{S}$, and $g: X^{\prime} \rightarrow X$ is a submersion (whence $g \mid X^{\prime}$ is of constant rank).

(d) Any pair $\left(X^{\prime}, Y^{\prime}\right)$ of strata of $\mathcal{S}^{\prime}$ satisfies condition $a_{g}$ (which makes sense in view of (C) $)$.

In the case $P$ is a point, we will drop "over $P$ ". 
Proposition 11.2 (Thom's second isotopy lemma). If $g$ is a Thom mapping over $P$, then $g$ is locally trivial over $P$.

The proof of this requires new machinery. Let $\{T\}$ be a system control data for the stratification $\mathcal{S}$ of $S$. We need the notion of a system $\left\{T^{\prime}\right\}$ of control data over $\{T\}$ for the stratification $\mathcal{S}^{\prime}$ of $S^{\prime}$.

Caution. A system of control data over $\{T\}$ is not a system of control data as previously defined. If we were to require that a system of control data over $\{T\}$ also be a system of control data tout court then the fundamental existence theorem for control data over $\{T\}$ (Proposition 11.3, below) would not be true.

Definition. Suppose $g$ is a Thom mapping. A system $\left\{T^{\prime}\right\}$ of control data for $\mathcal{S}^{\prime}$ over $\{T\}$ is a family of tubular neighborhoods, indexed by $\mathcal{S}^{\prime}$, where $T_{X}^{\prime}$ is a tubular neighborhood of $X$ in $M^{\prime}$ with the following properties:

(a) If $X^{\prime}$ and $Y^{\prime}$ are strata of $\mathcal{S}^{\prime}$ and $X^{\prime}<Y^{\prime}$, then the commutation relation

$$
\pi_{X^{\prime}} \pi_{Y^{\prime}}(v)=\pi_{X^{\prime}}(v)
$$

holds for all $v$ for which both sides are defined, i.e., all $v \in\left|T_{X^{\prime}}\right| \cap\left|T_{Y^{\prime}}\right|$ such that $\pi_{Y^{\prime}}(v) \in\left|T_{X^{\prime}}\right|$.

Furthermore, if $g\left(X^{\prime}\right)$ and $g\left(Y^{\prime}\right)$ lie in the same stratum of $\mathcal{S}$, then the commutation relation

$$
\rho_{X^{\prime}} \pi_{Y^{\prime}}(v)=\rho_{X^{\prime}}(v)
$$

holds for all $v$ for which both sides of this equation are defined.

(b) If $X^{\prime}$ is a stratum of $\mathcal{S}^{\prime}$ and $X$ is a stratum of $\mathcal{S}$ which contains $g\left(X^{\prime}\right)$, then

$$
g \pi_{X^{\prime}}(v)=\pi_{X} g(v)
$$

for all $v$ for which both sides of this equation are defined, i.e., for all $v \in$ $\left|T_{X^{\prime}}\right| \cap g^{-1}\left|T_{X}\right|$.

Note that (a) is weaker than the commutation relation for control data in the case $g\left(X^{\prime}\right)$ and $g\left(Y^{\prime}\right)$ are not in the same stratum of $\mathcal{S}$.

Proposition 11.3. If $g$ is a Thom mapping then for any system $\{T\}$ of control data for $\mathcal{S}$ there exists a system $\left\{T^{\prime}\right\}$ of control data for $\mathcal{S}^{\prime}$ over $\{T\}$.

The proof of this is similar to the proof of the existence theorem for control data (Proposition 7.1). We will only outline it.

Proof (Outline): Let $\mathcal{S}_{k}^{\prime}$ be the family of all strata of $\mathcal{S}^{\prime}$ of dimension $\leq k$, and let $S_{k}^{\prime}$ denote the union of all strata in $\mathcal{S}_{k}^{\prime}$. We will show by induction on $k$ that the proposition is true for $\mathcal{S}_{k}^{\prime}$ and $S_{k}^{\prime}$ in place of $\mathcal{S}^{\prime}$ and $S^{\prime}$. This will suffice to prove the proposition.

The case $k=0$ is trivial. For the inductive step, we suppose that for each stratum $X^{\prime}$ of $\mathcal{S}^{\prime}$ of dimension $<k$, we are given a tubular neighborhood $T_{X^{\prime}}$ of $X^{\prime}$ and that this family of tubular neighborhoods satisfies conditions (a) and (b) above.

By shrinking the $T_{X^{\prime}}$ if necessary, we may suppose that if $X^{\prime}$ and $Y^{\prime}$ are strata of dimension $<k$ which are not comparable, then $\left|T_{X^{\prime}}\right| \cap\left|T_{Y^{\prime}}\right|=\varnothing$. To construct the $T_{X^{\prime}}$ on the strata of dimension $k$, we may do it one stratum at a time, since the relations (a) and (b) impose no conditions on pairs of strata of the same dimension. Let $X^{\prime}$ be a stratum of $\mathcal{S}^{\prime}$ of dimension $k$. 
We construct the tubular neighborhood $T_{X^{\prime}}$ in two steps as follows. For each $\ell \leq k$, we let $U_{\ell}^{\prime}$ denote the union of all $\left|T_{Y^{\prime}}\right|$ for $Y^{\prime}<X^{\prime}$ and $\operatorname{dim} Y^{\prime} \geq \ell$. We let $X_{\ell}^{\prime}=U_{\ell}^{\prime} \cap X^{\prime}$. In the first step, we construct a tubular neighborhood $T_{\ell}^{\prime}$ of $X_{\ell}^{\prime}$ by decreasing induction on $\ell$, shrinking various $T_{Y}^{\prime}$ where necessary.

This step is carried out in essentially the same way as the first step in the proof of Proposition 7.1. We start the induction at $\ell=k$, where there is nothing to prove. For the inductive step, we suppose $T_{\ell+1}^{\prime}$ has been constructed. We observe that to construct $T_{\ell}^{\prime}$ it is enough to construct $T_{\ell}^{\prime}$ on $\left|T_{Y^{\prime}}\right| \cap X^{\prime}$ for each stratum $Y^{\prime}<X^{\prime}$ of dimension $\ell$ separately. Then there are two cases.

Case 1. If $g\left(Y^{\prime}\right)$ and $g\left(X^{\prime}\right)$ are in the same stratum of $\mathcal{S}$, then the construction is carried in the same way as the corresponding construction in the proof of Proposition 7.1. In this way we define $T_{\ell}^{\prime}$ on $\left|T_{Y^{\prime}}\right| \cap X^{\prime}$ so that the commutation relations (a) hold. (Note that (b) follows from (国) and the induction hypothesis in this case.)

Case 2. In the case $g\left(Y^{\prime}\right)$ and $g\left(X^{\prime}\right)$ are not in the same stratum of $\mathcal{S}$, the proof must be modified. Let $X$ be the stratum which contains $g\left(X^{\prime}\right)$ and let $Y$ be the stratum which contains $g\left(Y^{\prime}\right)$. Then $Y<X$. By shrinking $\left|T_{Y^{\prime}}\right|$ if necessary, we may suppose that $g\left(\left|T_{Y^{\prime}}\right|\right) \subseteq\left|T_{Y}\right|$. Let

$$
V=\left(\left|T_{Y}\right| \cap X\right) \times_{Y} Y^{\prime}
$$

where the fibre product is taken with respect to the mappings

$$
\begin{gathered}
\pi_{Y}:\left|T_{Y}\right| \cap X \rightarrow Y, \\
g: Y^{\prime} \rightarrow Y .
\end{gathered}
$$

Then the mapping

$$
G=\left(g, \pi_{Y^{\prime}}\right):\left|T_{Y^{\prime}}\right| \cap X^{\prime} \rightarrow V
$$

is defined because the following commutes:

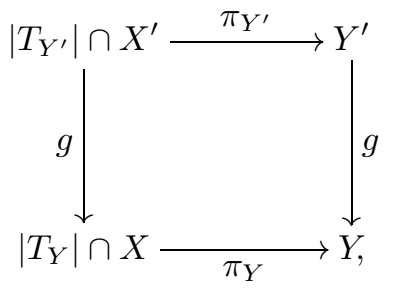

by the induction hypothesis that (b) is satisfied for those tubular neighborhoods which are already defined.

Lemma 11.4. There exists a neighborhood $N$ of $Y^{\prime}$ in $\left|T_{Y^{\prime}}\right|$ such that

$$
G \mid N \cap X^{\prime}: N \cap X^{\prime} \rightarrow V
$$

is a submersion.

Proof. Let $\Sigma$ be the set of points in $\left|T_{Y^{\prime}}\right| \cap X^{\prime}$ where the differential of $G$ is not onto. It suffices to show that $Y^{\prime} \cap \bar{\Sigma}=\varnothing$.

Let $x^{\prime} \in\left|T_{Y^{\prime}}\right| \cap X^{\prime}, x=g\left(x^{\prime}\right), y^{\prime}=\pi_{Y^{\prime}}\left(x^{\prime}\right)$, and $y=g\left(y^{\prime}\right)=\pi_{Y}(x)$. Then

$$
d G_{x^{\prime}}=\left(d\left(\pi_{Y^{\prime} X^{\prime}}\right)_{x^{\prime}}, d\left(g \mid X^{\prime}\right)_{x^{\prime}}\right): T X_{x^{\prime}}^{\prime} \rightarrow T V_{G\left(x^{\prime}\right)}=T X_{x} \times_{T Y_{y}} T Y_{y^{\prime}}^{\prime} .
$$

By definition, $x^{\prime} \in \Sigma$ if and only if this mapping is not onto. Since

$$
d\left(g \mid X^{\prime}\right)_{x^{\prime}}:: T X_{x^{\prime}}^{\prime} \rightarrow T X_{x}
$$

is onto (by hypothesis), it follows that this mapping is onto if and only if

$$
d\left(\pi_{Y^{\prime} X^{\prime}}\right)_{x^{\prime}}: \operatorname{ker}\left(d\left(g \mid X^{\prime}\right)_{x^{\prime}}\right) \rightarrow \operatorname{ker}\left(d\left(g \mid Y^{\prime}\right)_{y^{\prime}}\right)
$$


is onto. From condition $a_{g}$, it follows that $Y^{\prime}$ does not meet the closure $\bar{\Sigma}$ of the set of points where this mapping is not onto.

Now we extend $T_{\ell}^{\prime}$ over $\left|T_{Y^{\prime}}\right| \cap X^{\prime}$ in such a way that (国) holds (the weak (国)!) and (b) holds. We may do this by the generalized existence theorem for tubular neighborhoods and Lemma 11.4.

This completes the inductive step.

Now the second step (extension of $T_{\ell^{\prime}}$ from $U_{0}^{\prime}$ over all of $X^{\prime}$ ) is carried out in exactly the same way as in the proof of Proposition 7.1.

The rest of the proof of Proposition 11.2 will be carried out in three steps. First, we define the notion of a controlled vector field over another controlled vector field. (WARNING: this is not a special case of the notion of a controlled vector field.) Then we prove a lifting theorem for controlled vector fields. Finally, we show that every controlled vector field over another controlled vector field generates a local one-parameter group.

Now we suppose $g$ is a Thom mapping. We suppose that we are given a system $\{T\}$ of control data for $\mathcal{S}$ and a system $\left\{T^{\prime}\right\}$ of control data for $\mathcal{S}^{\prime}$ over $\{T\}$. Let $\eta=\left\{\eta_{X}\right\}_{X \in \mathcal{S}}$ be a controlled vector field on $S$.

Definition. By a controlled vector field on $S^{\prime}$ over $\eta$, we will mean a collection $\left\{\eta_{X^{\prime}}\right\}_{X^{\prime} \in \mathcal{S}^{\prime}}$ where $\eta_{X^{\prime}}$ is a vector field on $X^{\prime}$, such that the following conditions are satisfied.

(a) For any $X^{\prime} \in \mathcal{S}^{\prime}$ and $x^{\prime} \in X^{\prime}$, we have

$$
\left(g \mid X^{\prime}\right)_{*} \eta_{X^{\prime}}\left(x^{\prime}\right)=\eta_{X}\left(g\left(x^{\prime}\right)\right)
$$

where $X$ is the stratum of $\mathcal{S}$ that contains $g\left(x^{\prime}\right)$.

(b) For any $X^{\prime}, Y^{\prime} \in \mathcal{S}^{\prime}$ with $Y^{\prime}<X^{\prime}$, there is a neighborhood $N_{Y^{\prime}}$ of $Y^{\prime}$ in $\left|T_{Y^{\prime}}\right|$ such that for $y^{\prime} \in\left|T_{Y^{\prime}}\right| \cap X^{\prime}$, we have

$$
\left(\pi_{Y^{\prime}, X^{\prime}}\right)_{*} \eta_{X^{\prime}}\left(x^{\prime}\right)=\eta_{Y^{\prime}}\left(\pi_{Y^{\prime}, X^{\prime}}\left(x^{\prime}\right)\right)
$$

and if $g\left(X^{\prime}\right)$ and $g\left(Y^{\prime}\right)$ are in the same stratum of $\mathcal{S}$ then we have

$$
\eta_{X^{\prime}} \rho_{Y^{\prime}, X^{\prime}}\left(x^{\prime}\right)=0 \text {. }
$$

(Note that condition $\mathrm{b}$ is weaker than the condition that we imposed on a controlled vector field in Section 9 in the case $g\left(Y^{\prime}\right)$ and $g\left(X^{\prime}\right)$ are not in the same stratum of $\mathcal{S}$.)

Proposition 11.5. There exists a controlled vector field on $S^{\prime}$ over $\eta$. it.

The proof is completely analogous to the proof of Proposition 9.1 and we omit

Proposition 11.6. If $\eta^{\prime}$ is a controlled vector field on $S^{\prime}$ over $\eta$, then $\eta^{\prime}$ generates a local one parameter group, which commutes with the one-parameter group on $S$ generated by $\eta$.

The proof of this is essentially the same as the proof of Proposition 10.1, The only additional remark to be made is that if $X^{\prime}$ and $Y^{\prime}$ are strata of $\mathcal{S}$ with $Y^{\prime}<X^{\prime}$, and $g\left(Y^{\prime}\right)$ lies in $Y$ and $g\left(X^{\prime}\right)$ lies in $X$, then, in the case $Y<X$, a trajectory $y^{\prime}$ of $\eta^{\prime}$ starting at a point of $X^{\prime}$ cannot approach $Y^{\prime}$ because the image of $y^{\prime}$ is a trajectory of $\eta$ and therefore cannot approach a point of $Y$.

We omit the proof. 
Proof of Proposition 11.2. To prove that $g$ is locally trivial over $P$, it suffices to consider the case $P=\mathbb{R}^{p}$ and prove that $g$ is trivial over $P$ in this case. By Proposition 7.1 we can find a system $\{T\}$ of control data for $\mathcal{S}$ compatible with $f$, and by Proposition 11.3 there exists a system $\left\{T^{\prime}\right\}$ of control data for $\mathcal{S}^{\prime}$ over $\{T\}$. Let $\partial_{1}, \cdots, \partial_{p}$ be the coordinate vector fields on $\mathbb{R}^{p}$. By Proposition 9.1, we can lift $\partial_{i}$ to a controlled vector field $\widetilde{\partial}_{i}$ on $S$, and by Proposition 11.5 we can lift $\widetilde{\partial}_{i}$ to a controlled vector field $\widetilde{\widetilde{\partial}}_{i}$ on $S^{\prime}$ over $\widetilde{\partial}_{i}$.

By Propositions 10.1 and 11.6 the vector fields $\widetilde{\partial}_{i}$ and $\widetilde{\widetilde{\partial}}_{i}$ generate local oneparameter groups $\widetilde{\varphi}_{i}$ and $\widetilde{\widetilde{\varphi}}_{i}$. Since the mappings $f$ and $g$ are proper and $\partial_{i}$ generates a (global) one-parameter group $\varphi_{i}$, it follows that $\widetilde{\varphi}_{i}$ and $\widetilde{\varphi}_{i}$ are (global) one-parameter groups.

Let $S_{0}$ (resp. $S_{0}^{\prime}$ ) denote the fibre of $S$ (resp. $S^{\prime}$ ) over 0 . To complete the proof, it is enough to construct local homeomorphisms $h$ and $h^{\prime}$ such that the following diagram commutes.

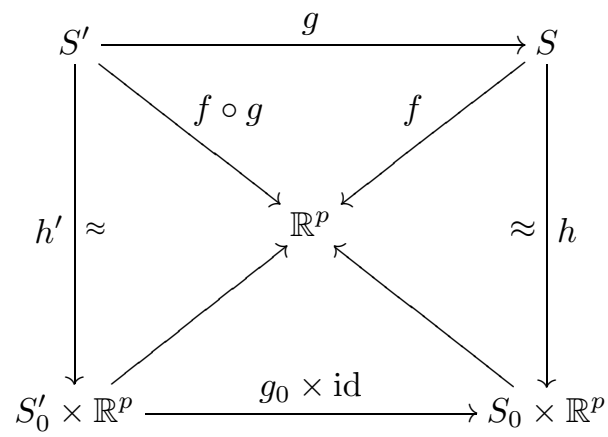

We define $h$ and $h^{\prime}$ as follows.

$$
\begin{aligned}
& h^{\prime}(x)=\left(\varphi_{p,-t_{p}}^{\prime} \cdots \varphi_{1,-t_{1}}^{\prime}(x), t\right) \quad \text { where } t=\left(t_{1}, \cdots, t_{p}\right)=f \circ g(x) \text {, } \\
& h(x)=\left(\varphi_{p,-t_{p}} \cdots \varphi_{1,-t_{1}}(x), t\right) \quad \text { where } t=\left(t_{1}, \cdots, t_{p}\right)=f(x) .
\end{aligned}
$$

It is easily verified that the above diagram commutes and that $h$ and $h^{\prime}$ are homeomorphisms.

\section{REFERENCES}

[1] J. Kelly, General Topology, Van Nostrand Co., Inc., Princeton, N.J., 1965.

[2] S. Lang, Introduction to differentiable manifolds, Interscience, New York, 1962. MR.0155257 $(27: 5192)$

[3] R. Thom, Local topological properties of differentiable mappings, "Colloquium on Differential Analysis". pp. 191-202, Tata Inst./Oxford Univ. Press, Bombay/London and New York, 1964. MR0195102 (33:3307)

[4] Ensembles et morphismes stratifiés, Bull. Amer. Math. Soc. 75 (1969), 240-284. MR0239613 (39:970)

[5] H. Whitney, Tangents to an analytic variety, Ann, of Math. 81 (1964), 496-549. MR0192520 $(33: 745)$

[6] Local properties of analytic varieties, Differential and Combinatorial Topology, Princeton Univ. Press, Princeton, 1965. 


\section{LisT OF REVISIONS}

The above article is a slightly revised version of the booklet that I wrote in 1970 . Here I describe the main changes.

There are a few places where I wrote something other than what I meant. The most important is the formulation of Proposition 7.1. I have changed "If $f: M \rightarrow P$ is a submersion" in the original to "If $f: M \rightarrow P$ is smooth and $f \mid X$ is a submersion of $X$ into $P$ for each stratum $X$."

I have changed "Let $\mu$ be a positive number" to "Let $\mu$ be a positive integer" in the second paragraph in Section 1.

In Example 2.5, I have changed "If $\left\{x_{i}\right\} \subset X$ is a sequence converging to 0, then the tangents $\left\{T X_{x_{i}}\right\}$ converge to a line $\tau \subseteq \mathbb{R}^{2}$, and $\widetilde{0 x_{i}}$ converges to a line $\ell$, which makes an angle $\alpha$ with $\tau$." to "If $\left\{x_{i}\right\} \subset X$ is a sequence converging to 0 and the tangents $T X_{x_{i}}$ converge to a line $\tau \subseteq \mathbb{R}^{2}$, then $\widehat{0 x}_{i}$ converges to a line $\ell$, which makes an angle $\alpha$ with $\tau$."

I have changed " $X=\bar{Y}=Y \cup X$ " following the remark in section 5 to " $X \subset$ $\bar{Y}=Y \cup X$."

I have changed " $\{x \in M: t \in I, H(x, t) \neq x\}$ " to " $\{x \in M: \exists t \in I, \quad H(x, t) \neq$ $x\}$ " in Section 6 .

I have changed "with support in $V$ " in the statement of Proposition 6.1 to "with support in $V-U^{\prime}$." I have added the hypothesis, "Suppose $\mu \geq 2 . "$

In the second paragraph of step 1 of the proof of Proposition 6.1, I have changed " $W_{i}$ is contained in $W_{x_{i}}$ " to " $W_{i}$ is open in $M$ and is contained in $W_{x_{i}}$ " In the sixth paragraph, I have changed " $U_{i-1}^{*} \subset W^{0}$ " to " $\bar{U}_{i}^{*} \subset U_{i-1}^{*} \cup W_{i}^{0}$."

I have added "(in the $C^{\mu-1}$ topology)" in the next to last paragraph before the lemma used in the proof of Proposition 6.1.

In the lemma used in the proof of Proposition 6.1, I have changed "inner products $i$ and $j$ " to "inner products $k$ and $l$ " and " $H \circ L: V \rightarrow V$ " to " $H \circ L: V \rightarrow W$."

In the existence part of the proof of this lemma, I have changed " $\left(L e_{i}, L e_{j}\right)_{j}$ " to " $\left(L e_{i}, L e_{j}\right) \ell$ " and "Then $f_{1}, \ldots, f_{n}$ is an orthonormal basis of $V$." to "Then $f_{1}, \ldots, f_{n}$ is an orthonormal basis of $W$."

I have changed " $\beta$ " to " $\beta_{1}$ " in both of the first two displayed formulas following the proof of this lemma. I have changed "close" to " $C^{1}$-close" immediately preceding the first of these formulas.

I have changed " $U_{1}$ " to " $U$ " " at the end of the first paragraph following the proof of this lemma.

I have changed " $g_{s}\left(V_{2}\right) \subseteq g_{t}\left(V_{1}\right)$ for $0 \leq s, t \leq 1$ " to " $V_{2} \subset g_{t}\left(V_{1}\right)$ for $0 \leq t \leq 1$ " in the second paragraph following the proof of this lemma.

Towards the end of the proof of Proposition 6.1, I have changed " $H$ " to " $\widetilde{H}$ " every time " $H$ " appears, " $g_{1}$ " to " $g_{1} \circ g_{0}^{-1}$ " (twice), " $H_{1} \circ \varphi$ " to " $\widetilde{H}_{1} \circ g_{0} \circ \varphi_{0}$ " and $H_{1 *}$ to $\left(\widetilde{H}_{1} g_{0}\right)_{*}$. The last paragraph in this proof is new.

In the proof of Proposition 6.2, third paragraph, I have changed "Furthermore, we can choose closed sets such that $W_{i}^{\prime} \subseteq W_{i}$ such that $\left\{W_{i}^{\prime}\right\}$ is a cover" to "Furthermore we can choose closed sets $W_{i}^{\prime}$ of $X$ such that $W_{i}^{\prime} \subset W_{i}$ and $\left\{W_{i}^{\prime}\right\}$ is a cover."

In the proof of Proposition 6.2, second paragraph from the end, I have changed " $U_{i}^{\prime \prime} \subseteq W_{i}-U_{i-1}^{\prime}$ " to " $\bar{U}_{i}^{\prime \prime} \subseteq W_{i} \cup U_{i-1}^{\prime \prime}$." 
Immediately before Lemma 7.3, I have changed "tubular restriction $\pi_{T}$ " to "projection $\pi_{T} . "$

In Section 8, before Definition 8.2, I have changed " $v \in T_{Y, Z}$ " to " $v \in T_{X, Z} \cap$ $T_{Y, Z}$."

In Section 8, fifth paragraph before the end, I have changed "and let $\pi_{X}: T_{X}^{\prime} \rightarrow$ $X$ and $\rho_{X}: T_{X}^{\prime} \rightarrow(0, \infty)$ " to "Let $T_{X}=T_{X}^{\prime} \cap V, \quad \pi_{X}=\pi_{X}^{\prime} \mid T_{X}$, and $\rho_{X}=\rho_{X}^{\prime} \mid T_{X}$."

In Section 8, last paragraph, I have changed "if $(V, \mathcal{S}, J)$ is a Whiteny stratified set," to "if $\mathcal{S}$ is a Whitney stratification of a subset $V$ of a manifold,"

In Section 9 following (9.4), I have removed "by inclusion" from the phrase "The set $\mathcal{S}_{v}$ of strata $Y<X$ such that $v \in T_{Y}^{2}$ is totally ordered by inclusion."

In the following paragraph, I have put a comma in the sentence "For, either $Z=Y$ or $Z<Y$."

In the first displayed formula following this, I have added

$$
\begin{aligned}
& =\left(\pi_{Y, X}\right)_{*} \eta_{X}(\sigma) v_{Z, Y} \\
& =\eta_{Y}\left(\pi_{Y, X}(v)\right) \rho_{Z, Y}
\end{aligned}
$$

In Section 10, I have added "and $\alpha_{0}(v)=v$ for all $v \in V$ " at the end of the second sentence. I have added "and $\alpha(0, v)=v$ for all $v \in V$ " to the condition given in (a) of the first definition. I have added "possibly infinite at one or both ends." to the condition given in (b). I have changed "Since $\alpha$ is a one-parameter group" to "Since $\alpha$ is a local one-parameter group" following (c) of the second definition. I have changed "(Section 5)" to "(Section 9)" immediately preceding Propostion 10.1. I have changed "Proposition 10.1" to "Proposition 9.1" in the second sentence of the first paragraph following Diagram 10.1. In Corollary 10.4, I have changed "let $X$ be a closed subset of $M$ " to "let $S$ be a closed subset of $M$."

I have changed "a smooth local one-parameter group" to "a unique smooth local one-parameter group" in the first paragraph of the proof of Proposition 10.1 and "Uniqueness is obvious." to "Uniqueness is obvious since $\left.J_{X}, \alpha_{X}\right)$ is unique." in the second paragraph.

In Section 11, following the third diagram, I have changed the sentence "Local triviality of a mapping $f$ over a space $Z$ has a consequence which will be very important in what follows" to "Local triviality of a mapping $f$ over $Z$ has a consequence which is very important in our proof that topologically stable mappings are dense."

In Case 1 of the proof of Proposition 11.3, I have changed "(Note that (b) follows from (a) in this case.)" to "(Note that (b) follows from (a) and the induction hypothesis in this case.)"

In (a) of the definition following Lemma 11.4, I have added "where $X$ is the stratum of $S$ that contains $g\left(x^{\prime}\right)$."

In my 1970 notes, I wrote about "pre-stratifications" and "pre-stratified sets." I have eliminated the prefix "pre-" from all such expressions. In three places, I referred to non-existent "figures". I have eliminated these references.

The sections "Foreword to the 1970 Booklet", "List of Revisions", "Acknowledgments", and "Contents" at the beginning of this article have been added.

The other changes either are very minor and do not affect the meaning in any way, or have been inadvertantly overlooked. 